Article

\title{
The Complexity of Some Classes of Pyramid Graphs Created from a Gear Graph
}

\author{
Jia-Bao Liu ${ }^{1}$ (1) and Salama Nagy Daoud ${ }^{2,3, *(1)}$ \\ 1 School of Mathematics and Physics, Anhui Jianzhu University, Hefei 230601, China; liujiabaoad@163.com \\ 2 Department of Mathematics, Faculty of Science, Taibah University, Al-Madinah 41411, Saudi Arabia \\ 3 Department of Mathematics and Computer Science, Faculty of Science, Menoufia University, \\ Shebin El Kom 32511, Egypt \\ * Correspondence: sdaoud@taibahu.edu.sa; Tel.: +966-598914649
}

Received: 8 November 2018; Accepted: 23 November 2018; Published: 2 December 2018

\begin{abstract}
The methods of measuring the complexity (spanning trees) in a finite graph, a problem related to various areas of mathematics and physics, have been inspected by many mathematicians and physicists. In this work, we defined some classes of pyramid graphs created by a gear graph then we developed the Kirchhoff's matrix tree theorem method to produce explicit formulas for the complexity of these graphs, using linear algebra, matrix analysis techniques, and employing knowledge of Chebyshev polynomials. Finally, we gave some numerical results for the number of spanning trees of the studied graphs.
\end{abstract}

Keywords: complexity; Chebyshev polynomials; gear graph; pyramid graphs

MSC: 05C05, 05C50

\section{Introduction}

The graph theory is a theory that combines computer science and mathematics, which can solve considerable problems in several fields (telecom, social network, molecules, computer network, genetics, etc.) by designing graphs and facilitating them through idealistic cases such as the spanning trees, see [1-10].

A spanning tree of a finite connected graph $G$ is a maximal subset of the edges that contains no cycle, or equivalently a minimal subset of the edges that connects all the vertices. The history of enumerating the number of spanning trees $\tau(G)$ of a graph $G$ dates back to 1842 when the physicist Kirchhoff [11] offered the matrix tree theorem established on the determinants of a certain matrix gained from the Laplacian matrix $L$ defined by the difference between the degree matrix $D$ and adjacency matrix $A$, where $D$ is a diagonal matrix, $D=\operatorname{dig}\left(d_{1}, d_{2}, \ldots, d_{n}\right)$ corresponding to a graph $G$ with $n$ vertices that has the vertex degree of $d_{i}$ in the $i$ th position of a graph $G$ and $A$ is a matrix with rows and columns labeled by graph vertices, with a 1 or 0 in position $\left(u_{i}, u_{j}\right)$ according to whether $u_{i}$ and $u_{j}$ are adjacent or not. That is

$$
L_{i j}= \begin{cases}a_{i} & \text { if } i=j \\ -1 & \text { if } i \neq j \text { and } i \text { is adjacent to } j \\ 0 & \text { otherwise }\end{cases}
$$

where $a_{i}$ denotes the degree of the vertex $i$.

This method allows beneficial results for a graph comprising a small number of vertices, but is not feasible for large graphs. There is one more method for calculating $\tau(G)$. Let $\lambda_{1} \geq \lambda_{2} \geq \ldots \geq \lambda_{k}=0$ 
denote the eigenvalues of the matrix $L$ of a graph $G$ with $n$ vertices. "Kelmans" and "Chelnokov" [12] have derived that

$$
\tau(G)=\frac{1}{k} \prod_{i=1}^{k-1} \lambda_{k}
$$

One of the favorite methods of calculating the complexity is the contraction-deletion theorem. For any graph $G$, the complexity $\tau(G)$ of $G$ is equal to $\tau(G)=\tau(G-e)+\tau(G / e)$, where $e$ is any edge of $G$, and where $G-e$ is the deletion of $e$ from $G$, and $G / e$ is the contraction of $e$ in $G$. This gives a recursive method to calculate the complexity of a graph $[13,14]$.

Another important method is using electrically equivalent transformations of networks. Yilun Shang [15] derived a closed-form formula for the enumeration of spanning trees the subdivided-line graph of a simple connected graph using the theory of electrical networks.

Many works have conceived techniques to derive the number of spanning trees of a graph, some of which can be found at [16-18].

Now, we give the following Lemma:

Lemma 1 [19]. $\tau(G)=\frac{1}{k^{2}} \operatorname{det}\left(k I-D^{c}+A^{c}\right)$ where $A^{c}$ and $D^{c}$ are the adjacency and degree matrices of $G^{c}$, the complement of $G$, respectively, and $I$ is the $k \times k$ identity matrix.

The characteristic of this formula is to express $\tau(G)$ straightway as a determinant rather than in terms of cofactors as in Kirchhoff theorem or eigenvalues as in Kelmans and Chelnokov formula.

\section{Chebyshev Polynomial}

In this part we insert some relations regarding Chebyshev polynomials of the first and second types which we use in our calculations.

We start from their definitions, see Yuanping, et al. [20].

Let $A_{n}(x)$ be $n \times n$ matrix such that

$$
A_{n}(x)=\left(\begin{array}{ccccc}
2 x & -1 & 0 & \cdots & 0 \\
-1 & 2 x & -1 & \ddots & \vdots \\
0 & \ddots & \ddots & \ddots & 0 \\
\vdots & \ddots & \ddots & \ddots & -1 \\
0 & \cdots & 0 & -1 & 2 x
\end{array}\right) .
$$

Furthermore, we render that the Chebyshev polynomials of the first type are defined by

$$
T_{n}(x)=\cos \left(n \cos ^{-1} x\right)
$$

The Chebyshev polynomials of the second type are defined by

$$
U_{n-1}(x)=\frac{1}{n} \frac{d}{d x} T_{n}(x)=\frac{\sin \left(n \cos ^{-1} x\right)}{\sin \left(\cos ^{-1} x\right)}
$$

It is easily confirmed that

$$
U_{n}(x)-2 x U_{n-1}(x)+U_{n-2}(x)=0
$$

It can then be shown from this recursion that by expanding $\operatorname{det} A_{n}(x)$ one obtains

$$
U_{n}(x)=\operatorname{det}\left(A_{n}(x)\right), n \geq 1
$$

Moreover, by solving the recursion (3), one gets the straightforward formula 


$$
U_{n}(x)=\frac{\left(x+\sqrt{x^{2}-1}\right)^{n+1}-\left(x-{\sqrt{x^{2}-1}}^{n+1}\right.}{2{\sqrt{x^{2}-1}}^{n+1}}, n \geq 1,
$$

where the conformity is valid for all complex $x$ (except at $x= \pm 1$, where the function can be taken as the limit).

The definition of $U_{n}(x)$ easily yields its zeros and it can therefore be confirmed that

$$
U_{n-1}(x)=2^{n-1} \prod_{j=1}^{n-1}\left(x-\cos \frac{j \pi}{n}\right)
$$

One further notes that

$$
U_{n-1}(-x)=(-1)^{n-1} U_{n-1}(x)
$$

From Equations (6) and (7), we have:

$$
U_{n-1}^{2}(x)=4^{n-1} \prod_{j=1}^{n-1}\left(x^{2}-\cos ^{2} \frac{j \pi}{n}\right)
$$

Finally, straightforward manipulation of the above formula produces the following formula (9), which is highly beneficial to us later:

$$
U_{n-1}^{2}\left(\sqrt{\frac{x+2}{4}}\right)=\prod_{j=1}^{n-1}\left(x-2 \cos \frac{2 j \pi}{n}\right)
$$

Moreover, one can see that

$$
\begin{gathered}
U_{n-1}{ }^{2}(x)=\frac{1-T_{2 n}(x)}{2\left(1-x^{2}\right)}=\frac{1-T_{n}\left(2 x^{2}-1\right)}{2\left(1-x^{2}\right)} \\
T_{n}(x)=\frac{1}{2}\left[\left(x+\sqrt{x^{2}-1}\right)^{n}+\left(\left(x-\sqrt{x^{2}-1}\right)^{n}\right]\right.
\end{gathered}
$$

Now we introduce the following important two Lemmas.

Lemma 2 [21]. Let $B_{n}(x)$ be $n \times n$ Circulant matrix such that

$$
B_{n}(x)=\left(\begin{array}{cccccc}
x & 0 & 1 & \cdots & 1 & 0 \\
0 & \ddots & \ddots & \ddots & \ddots & 1 \\
1 & \ddots & \ddots & \ddots & \ddots & \vdots \\
\vdots & \ddots & \ddots & \ddots & \ddots & 1 \\
1 & \ddots & \ddots & \ddots & \ddots & 0 \\
0 & 1 & \cdots & 1 & 0 & x
\end{array}\right) .
$$

Then for $n \geq 3, x \geq 4$, one has

$$
\operatorname{det}\left(B_{n}(x)\right)=\frac{2(x+n-3)}{x-3}\left[T_{n}\left(\frac{x-1}{2}\right)-1\right] .
$$

Lemma 3 [22]. If $A \in F^{n \times n}, B \in F^{n \times m}, C \in F^{m \times n}$ and $D \in F^{m \times m}$. Suppose that $A$ and $D$ are nonsingular matrices, then:

$$
\operatorname{det}\left(\begin{array}{cc}
A & B \\
C & D
\end{array}\right)=\operatorname{det}\left(A-B D^{-1} C\right) \operatorname{det} D=\operatorname{det} A \operatorname{det}\left(D-C A^{-1} B\right) .
$$

This Lemma gives a type of symmetry for some matrices which simplify our calculations of the complexity of graphs studied in this paper. 


\section{Main Results}

Definition 1. The pyramid graph $A_{n}^{(m)}$ is the graph created from the gear graph $G_{m+1}$ with vertices $\left\{u_{0} ; u_{1}, u_{2}, \ldots, u_{m} ; w_{1}, w_{2}, \ldots, w_{m}\right\}$ and $m$ sets of vertices, say, $\left\{v_{1}^{1}, v_{2}^{1}, \ldots, v_{n}^{1}\right\},\left\{v_{1}^{2}, v_{2}^{2}, \ldots, v_{n}^{2}\right\}, \ldots,\left\{v_{1}^{m}, v_{2}^{m}, \ldots, v_{n}^{m}\right\}$, such that for all $i=1,2, \ldots, n$ the vertex $v_{i}^{j}$ is adjacent to $u_{j}$ and $u_{j+1}$, where $j=1,2, \ldots, m-1$, and $v_{i}^{m}$ is adjacent to $u_{1}$ and $u_{m}$. See Figure 1 .

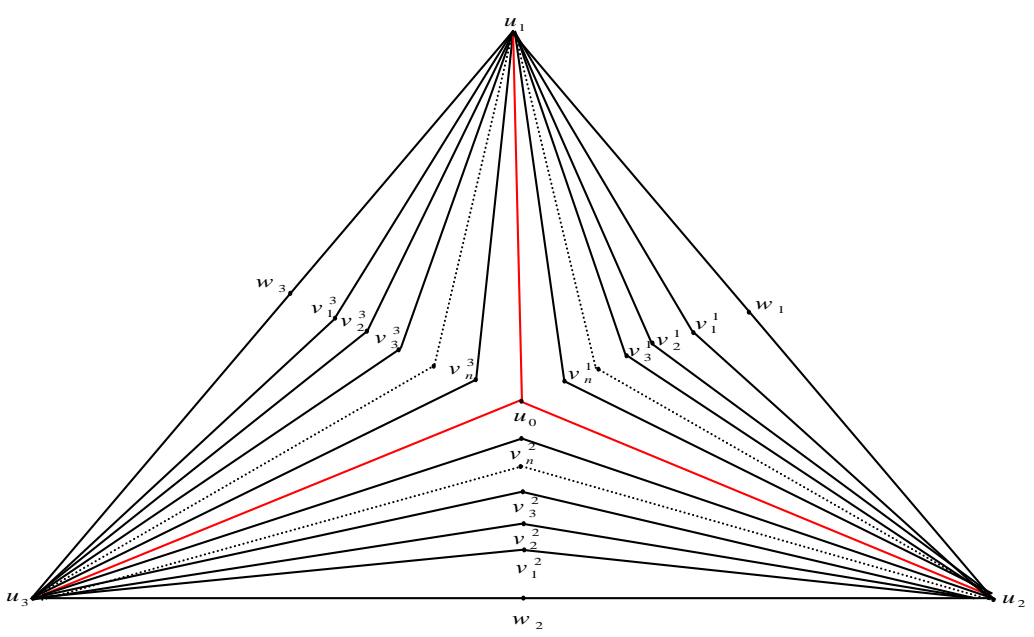

Figure 1. The pyramid graph $A_{n}^{(3)}$.

Theorem 1. For $n \geq 0, m \geq 3, \tau\left(A_{n}^{(m)}\right)=2^{m n}\left[(n+2+\sqrt{2 n+3})^{m}+(n+2-\sqrt{2 n+3})^{m}-\right.$ $\left.2(n+1)^{m}\right]$.

Proof. Using Lemma 1, we have

$$
\tau\left(A_{n}^{(m)}\right)=\frac{1}{(m n+2 m+1)^{2}} \times \operatorname{det}\left((m n+2 m+1) I-D^{c}+A^{c}\right)=\frac{1}{(m n+2 m+1)^{2}} \times
$$

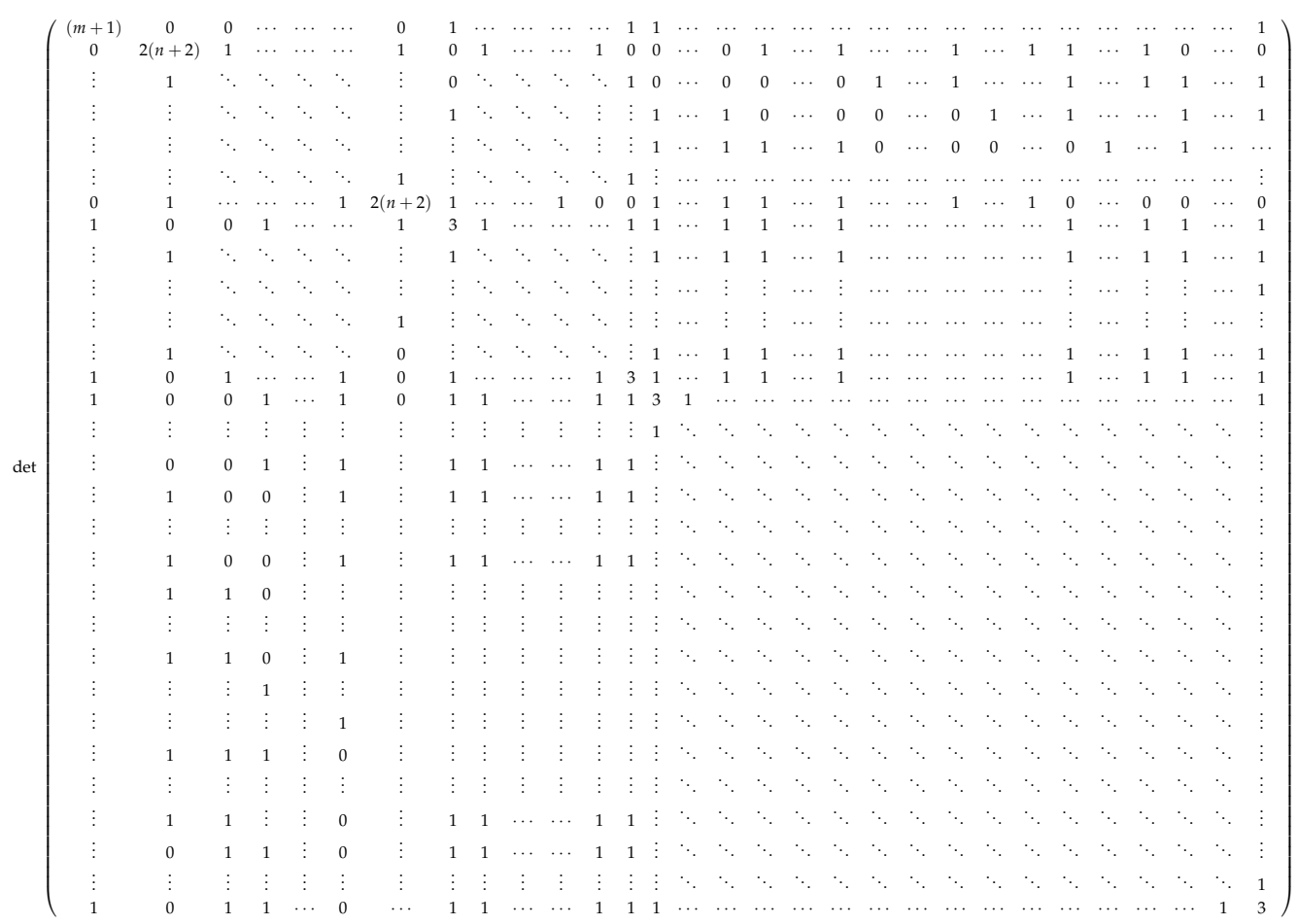


Let $j=(1 \cdots 1)$ be the $1 \times n$ matrix with all one, and $J_{n}$ be the $n \times n$ matrix with all one. Set $a=2 n+4$ and $b=m n+2 m+1$. Then we obtain:

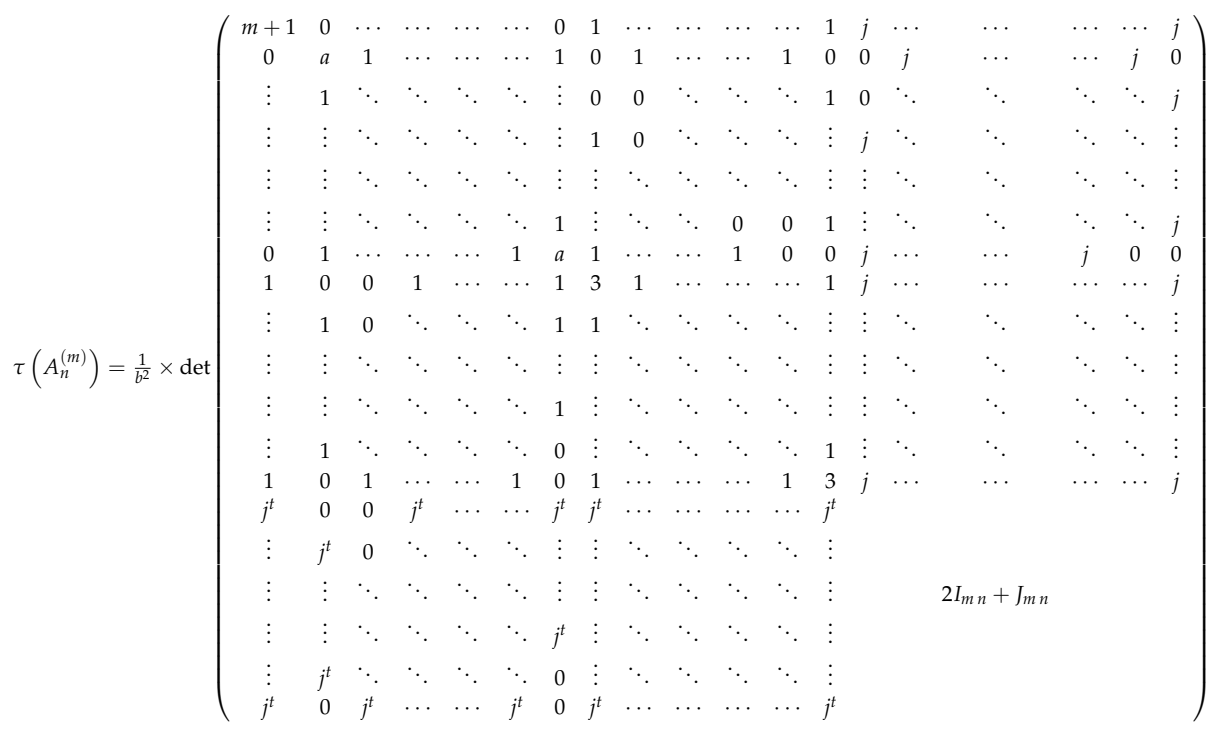

$$
\begin{aligned}
& =\frac{1}{b^{2}} \times \operatorname{det}\left(\begin{array}{ccccccccccccccccccccc}
b & 0 & \cdots & \cdots & \cdots & \cdots & 0 & 1 & \cdots & \cdots & \cdots & \cdots & 1 & j & \cdots & \cdots & \cdots & \cdots & j \\
b & a & 1 & \cdots & \cdots & \cdots & 1 & 0 & 1 & \cdots & \cdots & 1 & 0 & 0 & j & \cdots & \cdots & j & 0 \\
\vdots & 1 & \ddots & \ddots & \ddots & \ddots & \vdots & 0 & 0 & \ddots & \ddots & \ddots & 1 & 0 & \ddots & \ddots & \ddots & \ddots & j \\
\vdots & \vdots & \ddots & \ddots & \ddots & \ddots & \vdots & 1 & 0 & \ddots & \ddots & \ddots & \vdots & j & \ddots & \ddots & \ddots & \ddots & \vdots \\
\vdots & \vdots & \ddots & \ddots & \ddots & \ddots & \vdots & \vdots & \ddots & \ddots & \ddots & \ddots & \vdots & \vdots & \ddots & \ddots & \ddots & \ddots & \vdots \\
\vdots & \vdots & \ddots & \ddots & \ddots & \ddots & 1 & \vdots & \ddots & \ddots & 0 & 0 & 1 & \vdots & \ddots & \ddots & \ddots & \ddots & j \\
b & 1 & \cdots & \cdots & \cdots & 1 & a & 1 & \cdots & \cdots & 1 & 0 & 0 & j & \cdots & \cdots & j & 0 & 0 \\
b & 0 & 0 & 1 & \cdots & \cdots & 1 & 3 & 1 & \cdots & \cdots & \cdots & 1 & j & \cdots & \cdots & \cdots & \cdots & j \\
\vdots & 1 & 0 & \ddots & \ddots & \ddots & 1 & 1 & \ddots & \ddots & \ddots & \ddots & \vdots & \vdots & \ddots & & \ddots & \ddots & \ddots & \vdots \\
\vdots & \vdots & \ddots & \ddots & \ddots & \ddots & \vdots & \vdots & \ddots & \ddots & \ddots & \ddots & \vdots & \vdots & \ddots & & \ddots & \ddots & \ddots & \vdots \\
\vdots & \vdots & \ddots & \ddots & \ddots & \ddots & 1 & \vdots & \ddots & \ddots & \ddots & \ddots & \vdots & \vdots & \ddots & & \ddots & \ddots & \ddots & \vdots \\
\vdots & 1 & \ddots & \ddots & \ddots & \ddots & 0 & \vdots & \ddots & \ddots & \ddots & \ddots & 1 & \vdots & \ddots & & \ddots & \ddots & \ddots & \vdots \\
b & 0 & 1 & \cdots & \cdots & 1 & 0 & 1 & \cdots & \cdots & \cdots & 1 & 3 & j & \cdots & & \cdots & \cdots & \cdots & j \\
b j^{t} & 0 & 0 & j^{t} & \cdots & \cdots & j^{t} & j^{t} & \cdots & \cdots & \cdots & \cdots & j^{t} & & & & & & \vdots \\
\vdots & j^{t} & 0 & \ddots & \ddots & \ddots & \vdots & \vdots & \ddots & \ddots & \ddots & \ddots & \vdots & & & & & & \\
\vdots & \vdots & \ddots & \ddots & \ddots & \ddots & \vdots & \vdots & \ddots & \ddots & \ddots & \ddots & \vdots & & 2 I_{m n}+J_{m n} & & & \\
\vdots & \vdots & \ddots & \ddots & \ddots & \ddots & j^{t} & \vdots & \ddots & \ddots & \ddots & \ddots & \vdots & & & & & & \\
\vdots & j^{t} & \ddots & \ddots & \ddots & \ddots & 0 & \vdots & \ddots & \ddots & \ddots & \ddots & \vdots & & & & & & \\
b j^{t} & 0 & j^{t} & \cdots & \cdots & j^{t} & 0 & j^{t} & \cdots & \cdots & \cdots & \cdots & j^{t} & & & & & &
\end{array}\right) \\
& =\frac{1}{b} \times \operatorname{det}\left(\begin{array}{cccccccccccccccccccc}
1 & 0 & \cdots & \cdots & \cdots & \cdots & 0 & 1 & \cdots & \cdots & \cdots & \cdots & 1 & j & \cdots & \cdots & \cdots & \cdots & j \\
1 & a & 1 & \cdots & \cdots & \cdots & 1 & 0 & 1 & \cdots & \cdots & 1 & 0 & 0 & j & \cdots & \cdots & j & 0 \\
\vdots & 1 & \ddots & \ddots & \ddots & \ddots & \vdots & 0 & 0 & \ddots & \ddots & \ddots & 1 & 0 & \ddots & \ddots & \ddots & \ddots & j \\
\vdots & \vdots & \ddots & \ddots & \ddots & \ddots & \vdots & 1 & 0 & \ddots & \ddots & \ddots & \vdots & j & \ddots & & \ddots & \ddots & \ddots & \vdots \\
\vdots & \vdots & \ddots & \ddots & \ddots & \ddots & \vdots & \vdots & \ddots & \ddots & \ddots & \ddots & \vdots & \vdots & \ddots & & \ddots & \ddots & \ddots & \vdots \\
\vdots & \vdots & \ddots & \ddots & \ddots & \ddots & 1 & \vdots & \ddots & \ddots & 0 & 0 & 1 & \vdots & \ddots & \ddots & \ddots & \ddots & j \\
1 & 1 & \cdots & \cdots & \cdots & 1 & a & 1 & \cdots & \cdots & 1 & 0 & 0 & j & \cdots & \cdots & j & 0 & 0 \\
1 & 0 & 0 & 1 & \cdots & \cdots & 1 & 3 & 1 & \cdots & \cdots & \cdots & 1 & j & \cdots & \cdots & \cdots & \cdots & j \\
\vdots & 1 & 0 & \ddots & \ddots & \ddots & 1 & 1 & \ddots & \ddots & \ddots & \ddots & \vdots & \vdots & \ddots & \ddots & \ddots & \ddots & \vdots \\
\vdots & \vdots & \ddots & \ddots & \ddots & \ddots & \vdots & \vdots & \ddots & \ddots & \ddots & \ddots & \vdots & \vdots & \ddots & \ddots & \ddots & \ddots & \vdots \\
\vdots & \vdots & \ddots & \ddots & \ddots & \ddots & 1 & \vdots & \ddots & \ddots & \ddots & \ddots & \vdots & \vdots & \ddots & \ddots & \ddots & \ddots & \vdots \\
\vdots & 1 & \ddots & \ddots & \ddots & \ddots & 0 & \vdots & \ddots & \ddots & \ddots & \ddots & 1 & \vdots & \ddots & \ddots & \ddots & \ddots & \vdots \\
1 & 0 & 1 & \cdots & \cdots & 1 & 0 & 1 & \cdots & \cdots & \cdots & 1 & 3 & j & \cdots & \cdots & \cdots & \cdots & j \\
1 j^{t} & 0 & 0 & j^{t} & \cdots & \cdots & j^{t} & j^{t} & \cdots & \cdots & \cdots & \cdots & j^{t} & & & & & \\
\vdots & j^{t} & 0 & \ddots & \ddots & \ddots & \vdots & \vdots & \ddots & \ddots & \ddots & \ddots & \vdots & & & & & & \\
\vdots & \vdots & \ddots & \ddots & \ddots & \ddots & \vdots & \vdots & \ddots & \ddots & \ddots & \ddots & \vdots & & & 2 I_{m n}+J_{m n} & & & \\
\vdots & \vdots & \ddots & \ddots & \ddots & \ddots & j^{t} & \vdots & \ddots & \ddots & \ddots & \ddots & \vdots & & & & & & \\
\vdots & j^{t} & \ddots & \ddots & \ddots & \ddots & 0 & \vdots & \ddots & \ddots & \ddots & \ddots & \vdots & & & & & & \\
1 j^{t} & 0 & j^{t} & \cdots & \cdots & j^{t} & 0 & j^{t} & \cdots & \cdots & \cdots & \cdots & j^{t} & & & & & &
\end{array}\right)
\end{aligned}
$$



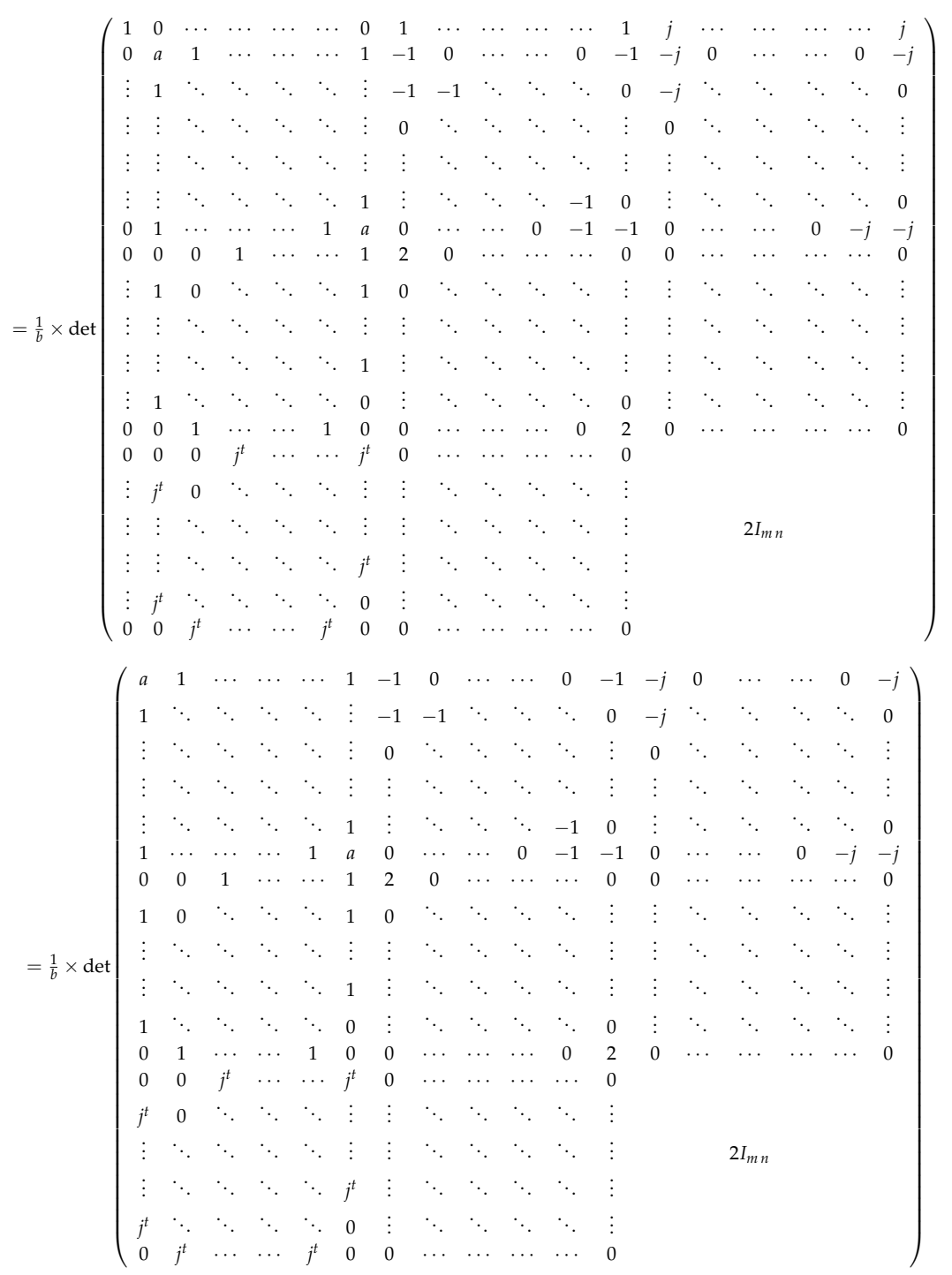

Using Lemma 3, yields

$$
\begin{aligned}
& \tau\left(A_{n}^{(m)}\right)=\frac{1}{b} \times \operatorname{det}\left(\begin{array}{cc}
A & B \\
C & 2 I_{m n}
\end{array}\right)=\frac{1}{b} \times \operatorname{det}\left(A-B \frac{1}{2 I_{m n}} C\right) \times 2^{m n} \\
& =\frac{1}{b} 2^{m n} \times 2^{-2 m} \times \operatorname{det}\left(\begin{array}{cccccccccccc}
2 a & n+2 & 2(n+1) & \cdots & 2(n+1) & n+2 & -2 & 0 & \cdots & \cdots & 0 & -2 \\
n+2 & 2 a & n+2 & 2(n+1) & \cdots & 2(n+1) & -2 & \ddots & \ddots & \ddots & \ddots & 0 \\
2(n+1) & n+2 & \ddots & \ddots & \ddots & \vdots & 0 & \ddots & \ddots & \ddots & \ddots & \vdots \\
\vdots & \ddots & \ddots & \ddots & \ddots & 2(n+1) & \vdots & \ddots & \ddots & \ddots & \ddots & \vdots \\
2(n+1) & \ddots & \ddots & \ddots & \ddots & n+2 & \vdots & \ddots & \ddots & \ddots & \ddots & 0 \\
n+2 & 2(n+1) & \cdots & 2(n+1) & n+2 & 2 a & 0 & \cdots & \cdots & 0 & -2 & -2 \\
0 & 0 & 2 & \cdots & \cdots & 2 & 4 & 0 & \cdots & \cdots & \cdots & 0 \\
2 & \ddots & \ddots & \ddots & \ddots & \vdots & 0 & \ddots & \ddots & \ddots & \ddots & \vdots \\
\vdots & \ddots & \ddots & \ddots & \ddots & \vdots & \vdots & \ddots & \ddots & \ddots & \ddots & \vdots \\
\vdots & \ddots & \ddots & \ddots & \ddots & 2 & \vdots & \ddots & \ddots & \ddots & \ddots & \vdots \\
2 & \ddots & \ddots & \ddots & \ddots & 0 & \vdots & \ddots & \ddots & \ddots & \ddots & 0 \\
0 & 2 & \cdots & \cdots & 2 & 0 & 0 & \cdots & \cdots & \cdots & 0 & 4
\end{array}\right)
\end{aligned}
$$

Using Lemma 3 again, yields

$$
\tau\left(A_{n}^{(m)}\right)=\frac{2^{m n-2 m}}{b} \times \operatorname{det}\left(\begin{array}{cc}
D & E \\
F & 4 I_{m}
\end{array}\right)=\frac{2^{m n}}{b} \times \operatorname{det}\left(D-E \frac{1}{4 I_{m}} F\right)
$$




$$
\tau\left(A_{n}^{(m)}\right)=\frac{2^{m n}}{b} \times \operatorname{det}\left(\begin{array}{cccccc}
2 a & (n+3) & 2(n+2) & \ldots & 2(n+2) & (n+3) \\
(n+3) & 2 a & (n+3) & \ddots & \ldots & 2(n+2) \\
2(n+2) & \ddots & \ddots & \ddots & \ddots & \vdots \\
\vdots & \ddots & \ddots & \ddots & \ddots & 2(n+2) \\
2(n+2) & \ddots & \ddots & \ddots & \ddots & (n+3) \\
(n+3) & 2(n+2) & \cdots & 2(n+2) & (n+3) & 2 a
\end{array}\right)
$$

Straightforward inducement using the properties of determinants, one can obtain

$$
\begin{aligned}
& \tau\left(A_{n}^{(m)}\right)=\frac{2^{m n}}{b} \times \frac{2 b}{m n+m+2} \times \operatorname{det}\left(\begin{array}{cccccc}
(2 a-n-3) & 0 & (n+1) & \cdots & (n+1) & 0 \\
0 & (2 a-n-3) & 0 & \ddots & \cdots & (n+1) \\
(n+1) & \ddots & \ddots & \ddots & \ddots & \vdots \\
\vdots & \ddots & \ddots & \ddots & \ddots & (n+1) \\
(n+1) & \ddots & \ddots & \ddots & \ddots & 0 \\
0 & (n+1) & \cdots & (n+1) & 0 & (2 a-n-3)
\end{array}\right) \\
& =\frac{2^{m n+1}(n+1)^{m}}{m n+m+2} \times \operatorname{det}\left(\begin{array}{cccccc}
\frac{(2 a-n-3)}{(n+1)} & 0 & 1 & \cdots & 1 & 0 \\
0 & \frac{(2 a-n-3)}{(n+1)} & 0 & \ddots & \ddots & 1 \\
1 & \ddots & \ddots & \ddots & \ddots & \vdots \\
\vdots & \ddots & \ddots & \ddots & \ddots & 1 \\
1 & \ddots & \ddots & \ddots & \ddots & 0 \\
0 & 1 & \cdots & 1 & 0 & \frac{(2 a-n-3)}{(n+1)}
\end{array}\right)
\end{aligned}
$$

Using Lemma 2, yields

$$
\begin{gathered}
\tau\left(A_{n}^{(m)}\right)=2^{m n+1} \times \frac{(n+1)^{m}}{m n+m+2} \times \frac{2\left(\frac{2 a-n-3}{n+1}+m-3\right)}{\frac{2 a-n-3}{n+1}-3} \times\left[T_{m}\left(\frac{\frac{2 a-n-3}{n+1}-1}{2}\right)-1\right] \\
=2^{m n+1} \times(n+1)^{m} \times\left[T_{m}\left(\frac{n+2}{n+1}\right)-1\right] .
\end{gathered}
$$

Using Equation (11), yields the result.

Definition 2. The pyramid graph $B_{n}^{(m)}$ is the graph created from the gear graph $G_{m+1}$ with vertices $\left\{u_{0} ; u_{1}, u_{2}, \ldots, u_{m} ; w_{1}, w_{2}, \ldots, w_{m}\right\}$ with double internal edges and $m$ sets of vertices, say, $\left\{v_{1}^{1}, v_{2}^{1}, \ldots, v_{n}^{1}\right\},\left\{v_{1}^{2}, v_{2}^{2}, \ldots, v_{n}^{2}\right\}, \ldots,\left\{v_{1}^{m}, v_{2}^{m}, \ldots, v_{n}^{m}\right\}$, such that for all $i=1,2, \ldots, n$ the vertex $v_{i}^{j}$ is adjacent to $u_{j}$ and $u_{j+1}$, where $j=1,2, \ldots, m-1$, and $v_{i}^{m}$ is adjacent to $u_{1}$ and $u_{m}$. See Figure 2 .

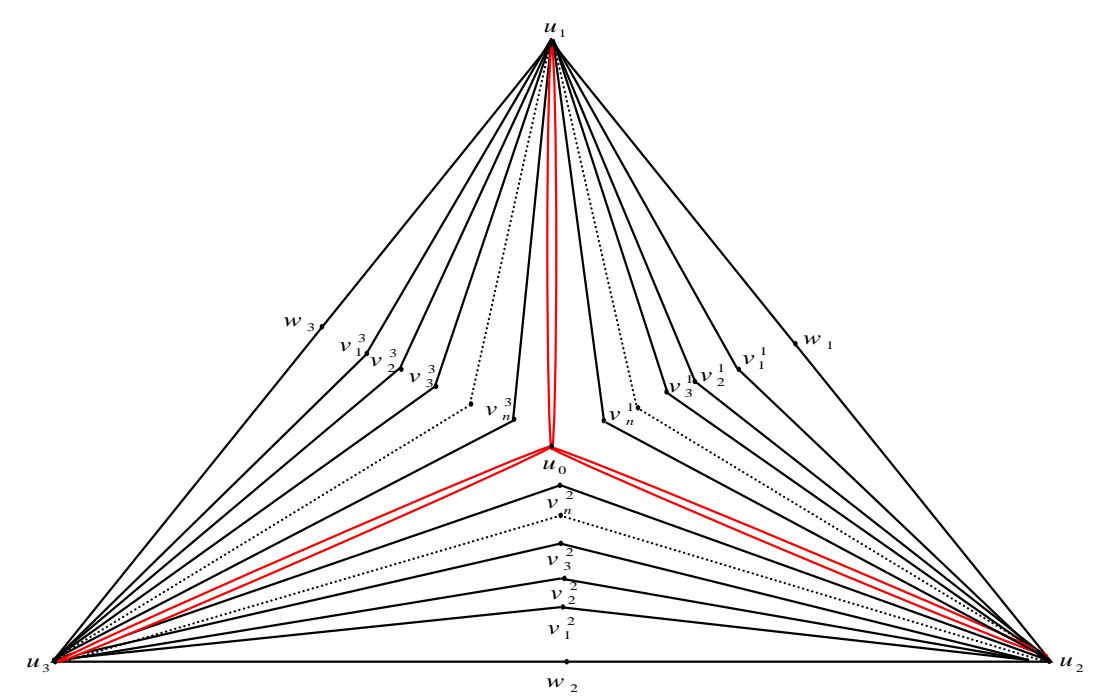

Figure 2. The pyramid graph $B_{n}^{(3)}$. 
Theorem 2. For $n \geq 0, m \geq 3, \tau\left(B_{n}^{(m)}\right)=2^{m n}\left[(n+3+2 \sqrt{n+2})^{m}+(n+3-2 \sqrt{n+2})^{m}-\right.$ $\left.2(n+1)^{m}\right]$.

Proof. Using Lemma 1, we get:

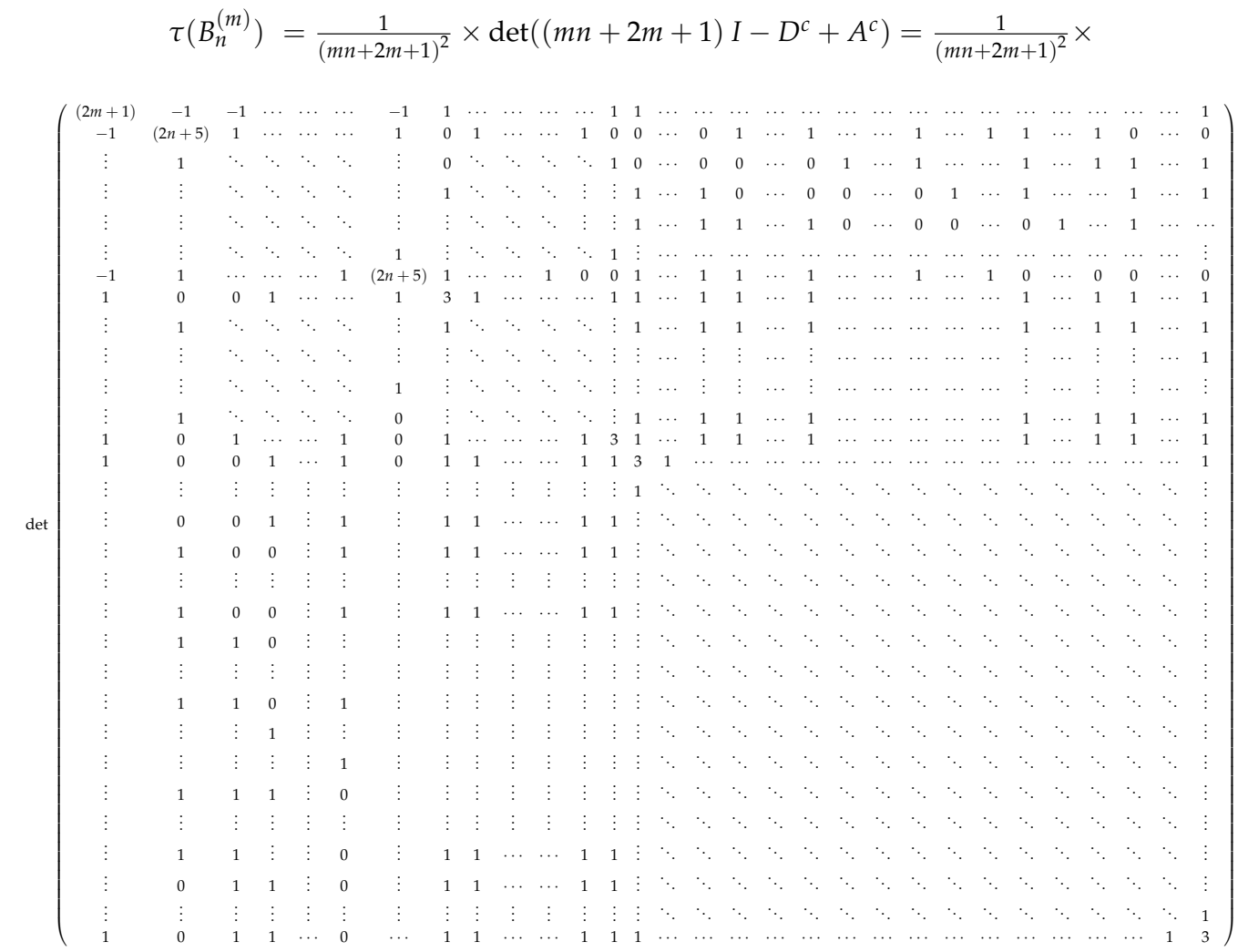

Let $j=(1 \cdots 1)$ be the $1 \times n$ matrix with all one, and $J_{n}$ be the $n \times n$ matrix with all one. Set $a=2 n+5$ and $b=m n+2 m+1$. Then we get:

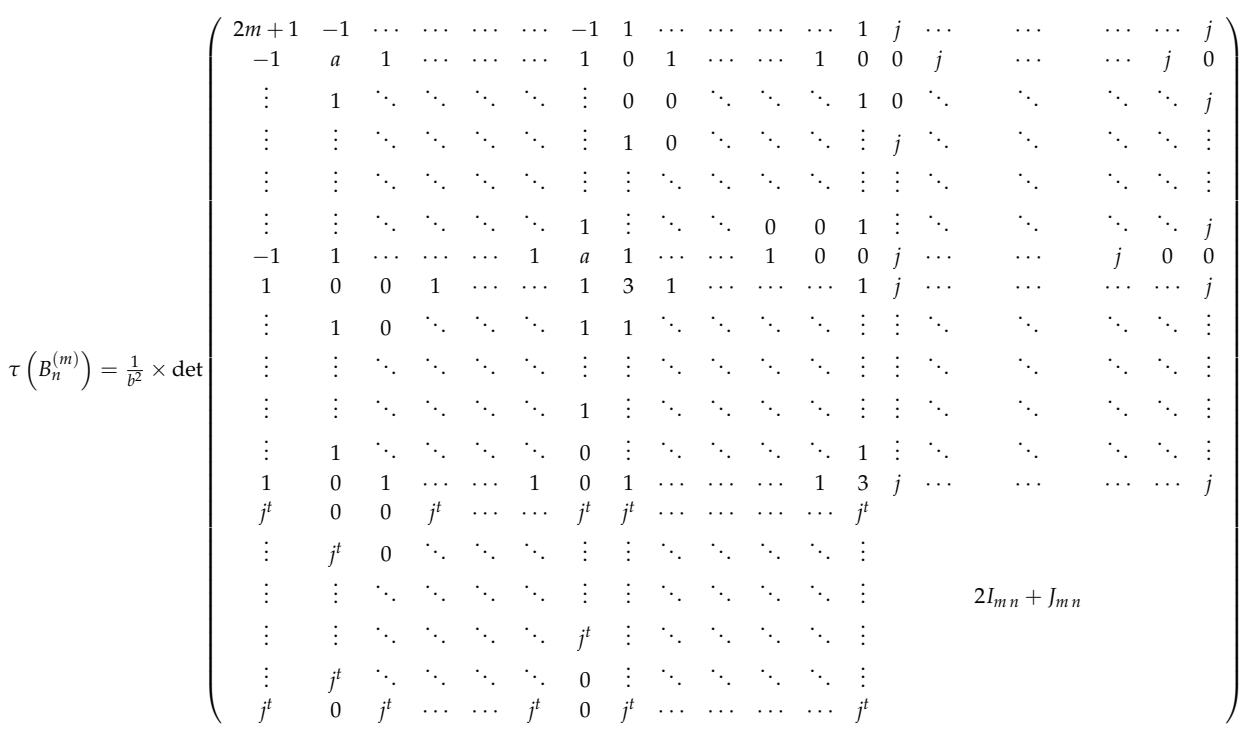




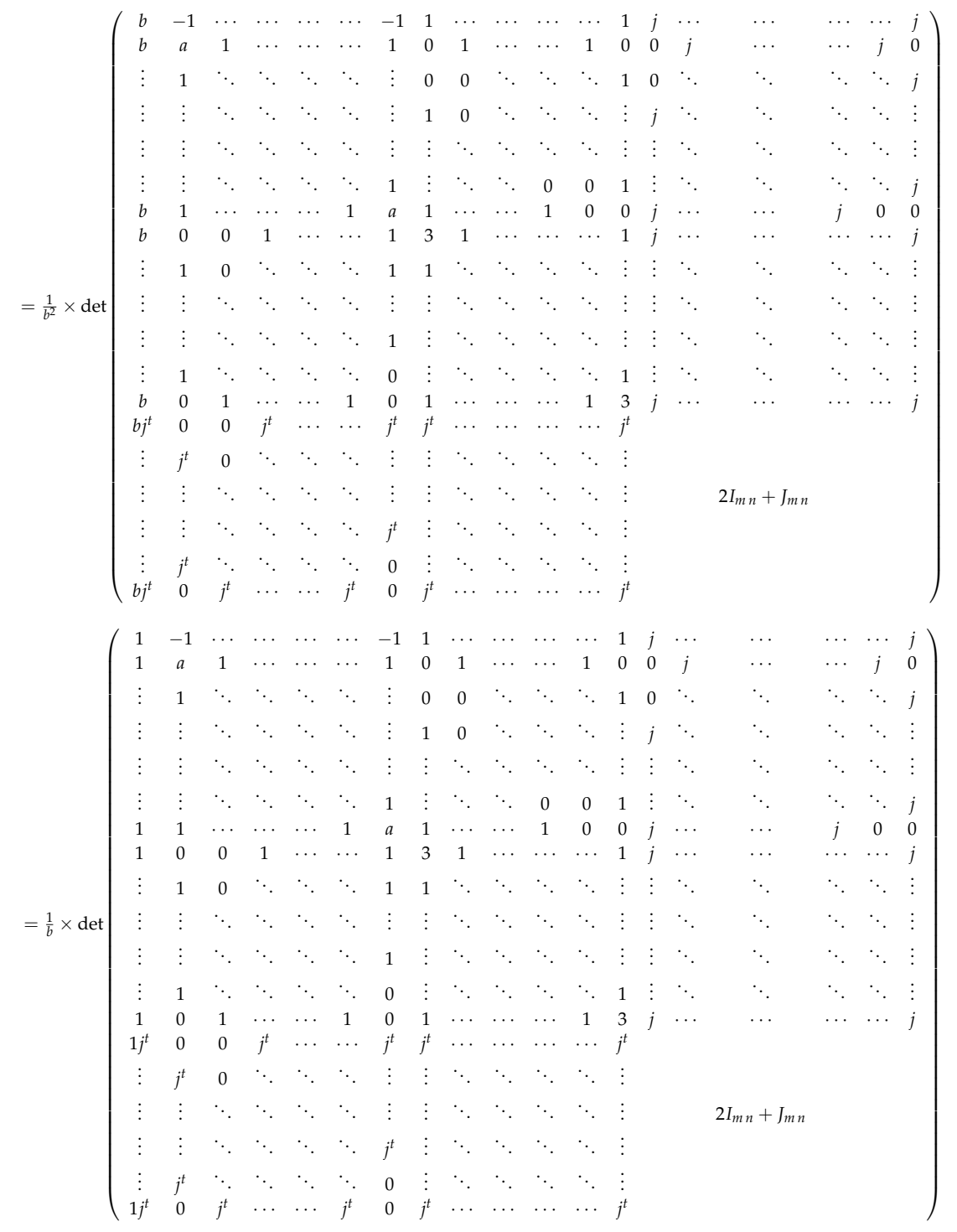

$=\frac{1}{b} \times \operatorname{det}\left(\begin{array}{ccccccccccccccccccc}1 & -1 & \cdots & \cdots & \cdots & \cdots & -1 & 1 & \cdots & \cdots & \cdots & \cdots & 1 & j & \cdots & \cdots & \cdots & \cdots & j \\ 0 & (a+1) & 2 & \cdots & \cdots & \cdots & 2 & -1 & 0 & \cdots & \cdots & 0 & -1 & -j & 0 & \cdots & \cdots & 0 & -j \\ \vdots & 2 & \ddots & \ddots & \ddots & \ddots & \vdots & -1 & -1 & \ddots & \ddots & \ddots & 0 & -j & \ddots & \ddots & \ddots & \ddots & 0 \\ \vdots & \vdots & \ddots & \ddots & \ddots & \ddots & \vdots & 0 & \ddots & \ddots & \ddots & \ddots & \vdots & 0 & \ddots & \ddots & \ddots & \ddots & \vdots \\ \vdots & \vdots & \ddots & \ddots & \ddots & \ddots & \vdots & \vdots & \ddots & \ddots & \ddots & \ddots & \vdots & \vdots & \ddots & \ddots & \ddots & \ddots & \vdots \\ \vdots & \vdots & \ddots & \ddots & \ddots & \ddots & 2 & \vdots & \ddots & \ddots & \ddots & -1 & 0 & \vdots & \ddots & \ddots & \ddots & \ddots & 0 \\ 0 & 2 & \cdots & \cdots & \cdots & 2 & (a+1) & 0 & \cdots & \cdots & 0 & -1 & -1 & 0 & \cdots & \cdots & 0 & -j & -j \\ 0 & 1 & 1 & 2 & \cdots & \cdots & 2 & 2 & 0 & \cdots & \cdots & \cdots & 0 & 0 & \cdots & \cdots & \cdots & \cdots & 0 \\ \vdots & 2 & 1 & \ddots & \ddots & \ddots & 2 & 0 & \ddots & \ddots & \ddots & \ddots & \vdots & \vdots & \ddots & \ddots & \ddots & \ddots & \vdots \\ \vdots & \vdots & \ddots & \ddots & \ddots & \ddots & \vdots & \vdots & \ddots & \ddots & \ddots & \ddots & \vdots & \vdots & \ddots & \ddots & \ddots & \ddots & \vdots \\ \vdots & \vdots & \ddots & \ddots & \ddots & \ddots & 2 & \vdots & \ddots & \ddots & \ddots & \ddots & \vdots & \vdots & \ddots & \ddots & \ddots & \ddots & \vdots \\ \vdots & 2 & \ddots & \ddots & \ddots & \ddots & 1 & \vdots & \ddots & \ddots & \ddots & \ddots & 0 & \vdots & \ddots & \ddots & \ddots & \ddots & \vdots \\ 0 & 1 & 2 & \cdots & \cdots & 2 & 1 & 0 & \cdots & \cdots & \cdots & 0 & 2 & 0 & \cdots & \cdots & \cdots & \cdots & 0 \\ 0 & j^{t} & j^{t} & 2 j^{t} & \cdots & \cdots & 2 j^{t} & 0 & \cdots & \cdots & \cdots & \cdots & 0 & & & & & \\ \vdots & 2 j^{t} & j^{t} & \ddots & \ddots & \ddots & \vdots & \vdots & \ddots & \ddots & \ddots & \ddots & \vdots & & & & & \\ \vdots & \vdots & \ddots & \ddots & \ddots & \ddots & \vdots & \vdots & \ddots & \ddots & \ddots & \ddots & \vdots & & & 2 I_{m n} & & \\ \vdots & \vdots & \ddots & \ddots & \ddots & \ddots & 2 j^{t} & \vdots & \ddots & \ddots & \ddots & \ddots & \vdots & & & & & \\ \vdots & 2 j^{t} & \ddots & \ddots & \ddots & \ddots & j^{t} & \vdots & \ddots & \ddots & \ddots & \ddots & \vdots & & & & & \\ 0 & j^{t} & 2 j^{t} & \cdots & \cdots & 2 j^{t} & j^{t} & 0 & \cdots & \cdots & \cdots & \cdots & 0 & & & & & \end{array}\right)$ 


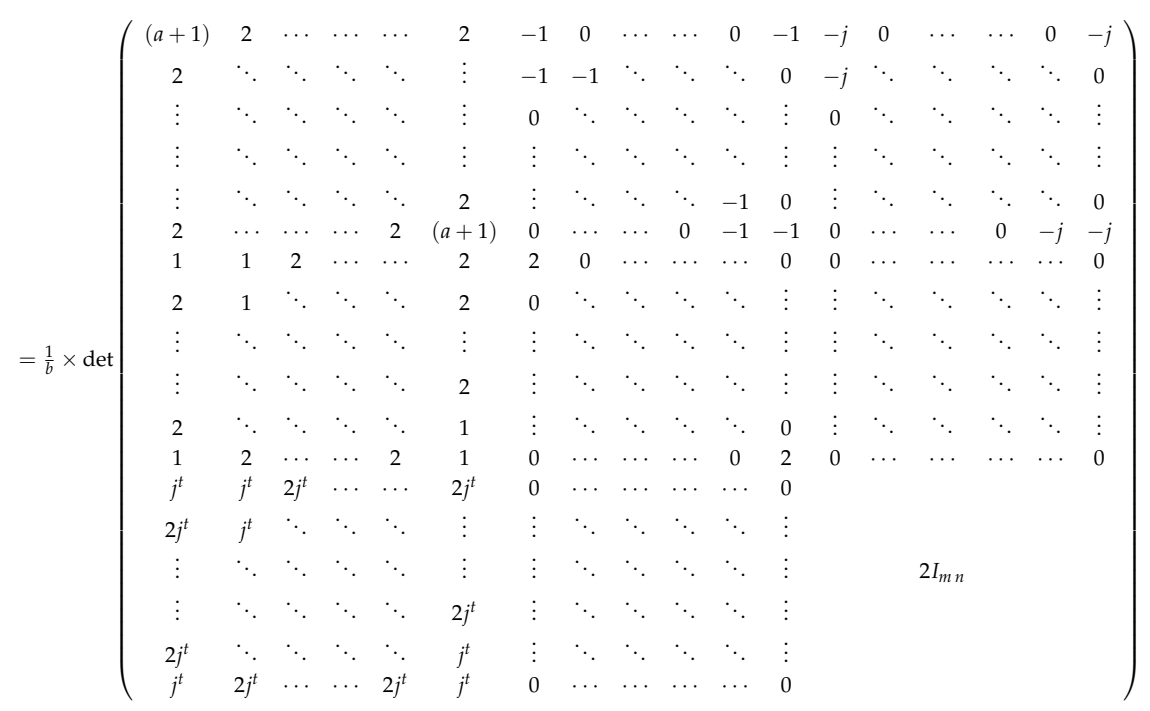

Using Lemma 3, yields

$$
\begin{aligned}
& \tau\left(B_{n}^{(m)}\right)=\frac{1}{b} \times \operatorname{det}\left(\begin{array}{cc}
A & B \\
C & 2 I_{m n}
\end{array}\right)=\frac{1}{b} \times \operatorname{det}\left(A-B \frac{1}{2 I_{m n}} C\right) \times 2^{m n} \\
& =\frac{1}{5} 2^{m n} \times 2^{-2 m} \times \operatorname{det}\left(\begin{array}{cccccccccccc}
(2 a+2 n+2) & 3 n+4 & 4(n+1) & \cdots & 4(n+1) & 3 n+4 & -2 & 0 & \cdots & \cdots & 0 & -2 \\
3 n+4 & (2 a+2 n+2) & 3 n+4 & 4(n+1) & \cdots & 4(n+1) & -2 & \ddots & \ddots & \ddots & \ddots & 0 \\
4(n+1) & 3 n+4 & \ddots & \ddots & \ddots & \vdots & 0 & \ddots & \ddots & \ddots & \ddots & \vdots \\
\vdots & \ddots & \ddots & \ddots & \ddots & 4(n+1) & \vdots & \ddots & \ddots & \ddots & \ddots & \vdots \\
4(n+1) & \ddots & \ddots & \ddots & \ddots & 3 n+4 & \vdots & \ddots & \ddots & \ddots & \ddots & 0 \\
3 n+4 & 4(n+1) & \cdots & 4(n+1) & 3 n+4 & (2 a+2 n+2) & 0 & \cdots & \cdots & 0 & -2 & -2 \\
2 & 2 & 4 & \cdots & \cdots & 4 & 4 & 0 & \cdots & \cdots & \cdots & 0 \\
4 & \ddots & \ddots & \ddots & \ddots & \vdots & 0 & \ddots & \ddots & \ddots & \ddots & \vdots \\
\vdots & \ddots & \ddots & \ddots & \ddots & \vdots & \vdots & \ddots & \ddots & \ddots & \ddots & \vdots \\
\vdots & \ddots & \ddots & \ddots & \ddots & 4 & \vdots & \ddots & \ddots & \ddots & \ddots & \vdots \\
4 & \ddots & \ddots & \ddots & \ddots & 2 & \vdots & \ddots & \ddots & \ddots & \ddots & 0 \\
2 & 4 & \cdots & \cdots & 4 & 2 & 0 & \cdots & \cdots & \cdots & 0 & 4
\end{array}\right)
\end{aligned}
$$

Using Lemma 3 again, yields

$$
\begin{aligned}
& \tau\left(B_{n}^{(m)}\right)=\frac{2^{m n-2 m}}{b} \times \operatorname{det}\left(\begin{array}{cc}
D & E \\
F & 4 I_{m}
\end{array}\right)=\frac{2^{m n}}{b} \times \operatorname{det}\left(D-E \frac{1}{4 I_{m}} F\right) \\
& \tau\left(B_{n}^{(m)}\right)=\frac{2^{m n}}{b} \times \operatorname{det}\left(\begin{array}{cccccc}
(2 a+2 n+4) & (3 n+7) & 4(n+2) & \ldots & 4(n+2) & (3 n+7) \\
(3 n+7) & (2 a+2 n+4) & (3 n+7) & \ddots & \ldots & 4(n+2) \\
4(n+2) & \ddots & \ddots & \ddots & \ddots & \vdots \\
\vdots & \ddots & \ddots & \ddots & \ddots & 4(n+2) \\
4(n+2) & \ddots & \ddots & \ddots & \ddots & (3 n+7) \\
(3 n+7) & 4(n+2) & \ldots & 4(n+2) & (3 n+7) & (2 a+2 n+4)
\end{array}\right)
\end{aligned}
$$

With a straightforward inducement using properties of determinants, we obtain

$$
\tau\left(B_{n}^{(m)}\right)=\frac{2^{m n}}{b} \times \frac{4 b}{m n+m+4} \times \operatorname{det}\left(\begin{array}{cccccc}
(2 a-n-3) & 0 & (n+1) & \cdots & (n+1) & 0 \\
0 & (2 a-n-3) & 0 & \ddots & \ldots & (n+1) \\
(n+1) & \ddots & \ddots & \ddots & \ddots & \vdots \\
\vdots & \ddots & \ddots & \ddots & \ddots & (n+1) \\
(n+1) & \ddots & \ddots & \ddots & \ddots & 0 \\
0 & (n+1) & \cdots & (n+1) & 0 & (2 a-n-3)
\end{array}\right)
$$




$$
=\frac{2^{m n+2} \times(n+1)^{m}}{m n+m+4} \times \operatorname{det}\left(\begin{array}{cccccc}
\frac{(2 a-n-3)}{(n+1)} & 0 & 1 & \cdots & 1 & 0 \\
0 & \frac{(2 a-n-3)}{(n+1)} & 0 & \ddots & \ddots & 1 \\
1 & \ddots & \ddots & \ddots & \ddots & \vdots \\
\vdots & \ddots & \ddots & \ddots & \ddots & 1 \\
1 & \ddots & \ddots & \ddots & \ddots & 0 \\
0 & 1 & \cdots & 1 & 0 & \frac{(2 a-n-3)}{(n+1)}
\end{array}\right)
$$

Using Lemma 2, yields

$$
\begin{gathered}
\tau\left(B_{n}^{(m)}\right)=2^{m n+2} \times \frac{(n+1)^{m}}{m n+m+4} \times \frac{2\left(\frac{2 a-n-3}{n+1}+m-3\right)}{\frac{2 a-n-3}{n+1}-3} \times\left[T_{m}\left(\frac{\frac{2 a-n-3}{n+1}-1}{2}\right)-1\right] \\
=2^{m n+1} \times(n+1)^{m} \times\left[T_{m}\left(\frac{n+3}{n+1}\right)-1\right] .
\end{gathered}
$$

Using Equation (11), yields the result.

Definition 3. The pyramid graph $C_{n}^{(m)}$ is the graph created from the gear graph $G_{m+1}$ with vertices $\left\{u_{0} ; u_{1}, u_{2}, \ldots, u_{m} ; w_{1}, w_{2}, \ldots, w_{m}\right\}$ with double external edges and $m$ sets of vertices, say, $\left\{v_{1}^{1}, v_{2}^{1}, \ldots, v_{n}^{1}\right\},\left\{v_{1}^{2}, u_{2}^{2}, \ldots, v_{n}^{2}\right\}, \ldots,\left\{v_{1}^{m}, v_{2}^{m}, \ldots, v_{n}^{m}\right\}$, such that for all $i=1,2, \ldots, n$ the vertex $v_{i}^{j}$ is adjacent to $u_{j}$ and $u_{j+1}$, where $j=1,2, \ldots, m-1$, and $v_{i}^{m}$ is adjacent to $u_{1}$ and $u_{m}$. See Figure 3.

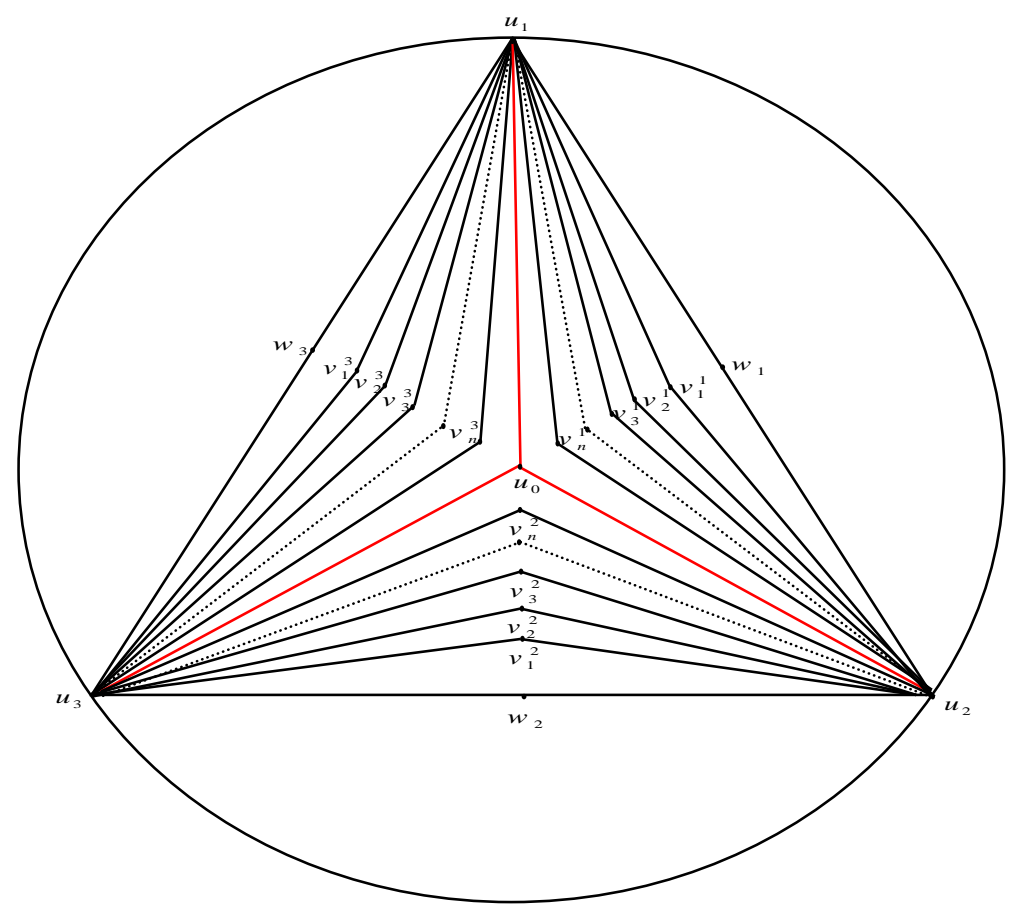

Figure 3. The pyramid graph $C_{n}^{(3)}$.

Theorem 3. For $n \geq 0, m \geq 3, \tau\left(C_{n}^{(m)}\right)=2^{m n}\left[(n+4+\sqrt{2 n+7})^{m}+(n+4-\sqrt{2 n+7})^{m}-\right.$ $\left.2(n+3)^{m}\right]$.

Proof. Using Lemma 1, we have:

$$
\tau\left(C_{n}^{(m)}\right)=\frac{1}{(m n+2 m+1)^{2}} \times \operatorname{det}\left((m n+2 m+1) I-D^{c}+A^{c}\right)=\frac{1}{(m n+2 m+1)^{2}} \times
$$




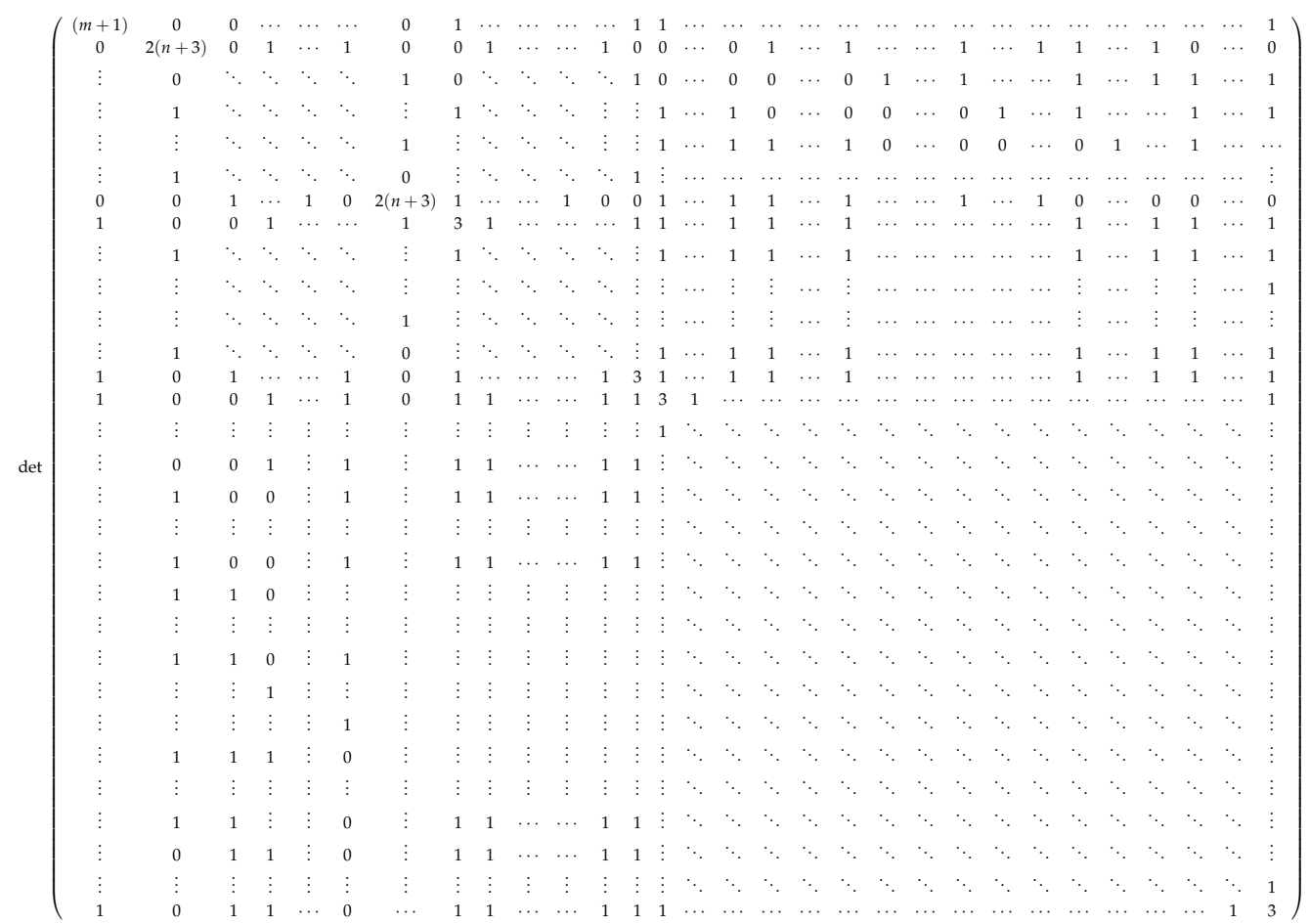

Let $j=(1 \cdots 1)$ be the $1 \times n$ matrix with all one, and $J_{n}$ be the $n \times n$ matrix with all one. Set $a=2 n+6$ and $b=m n+2 m+1$. Then we have:

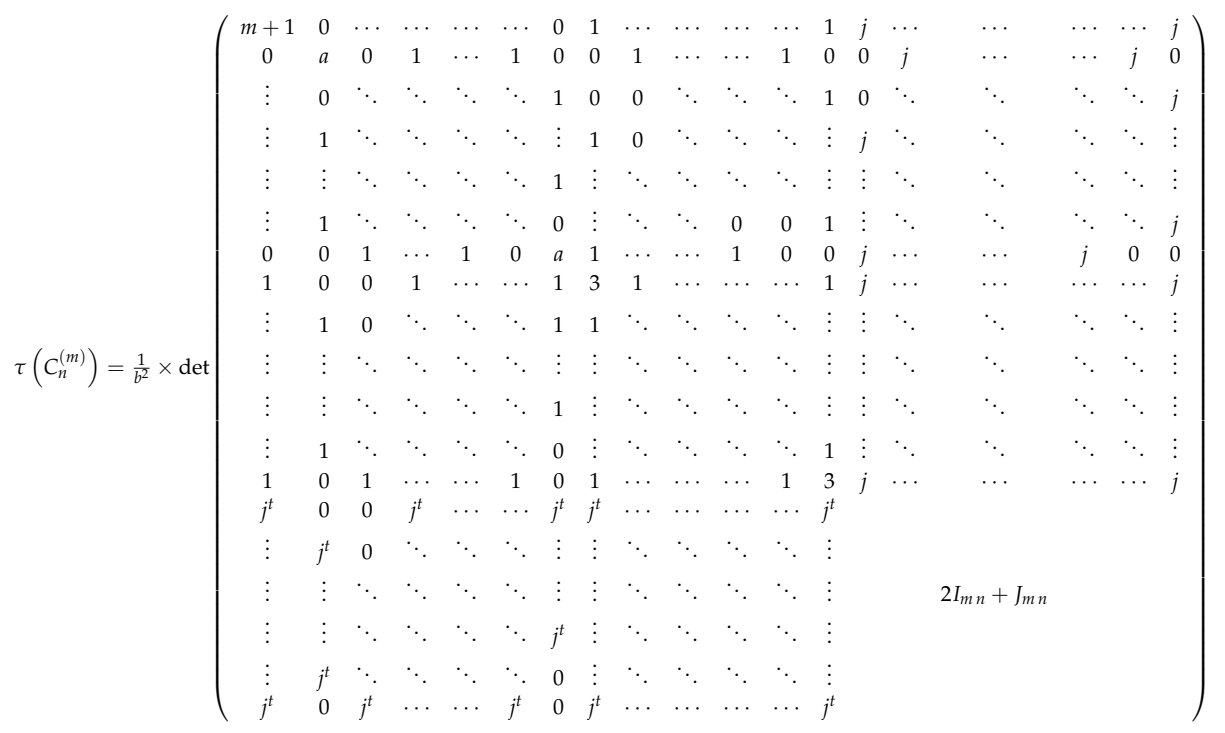



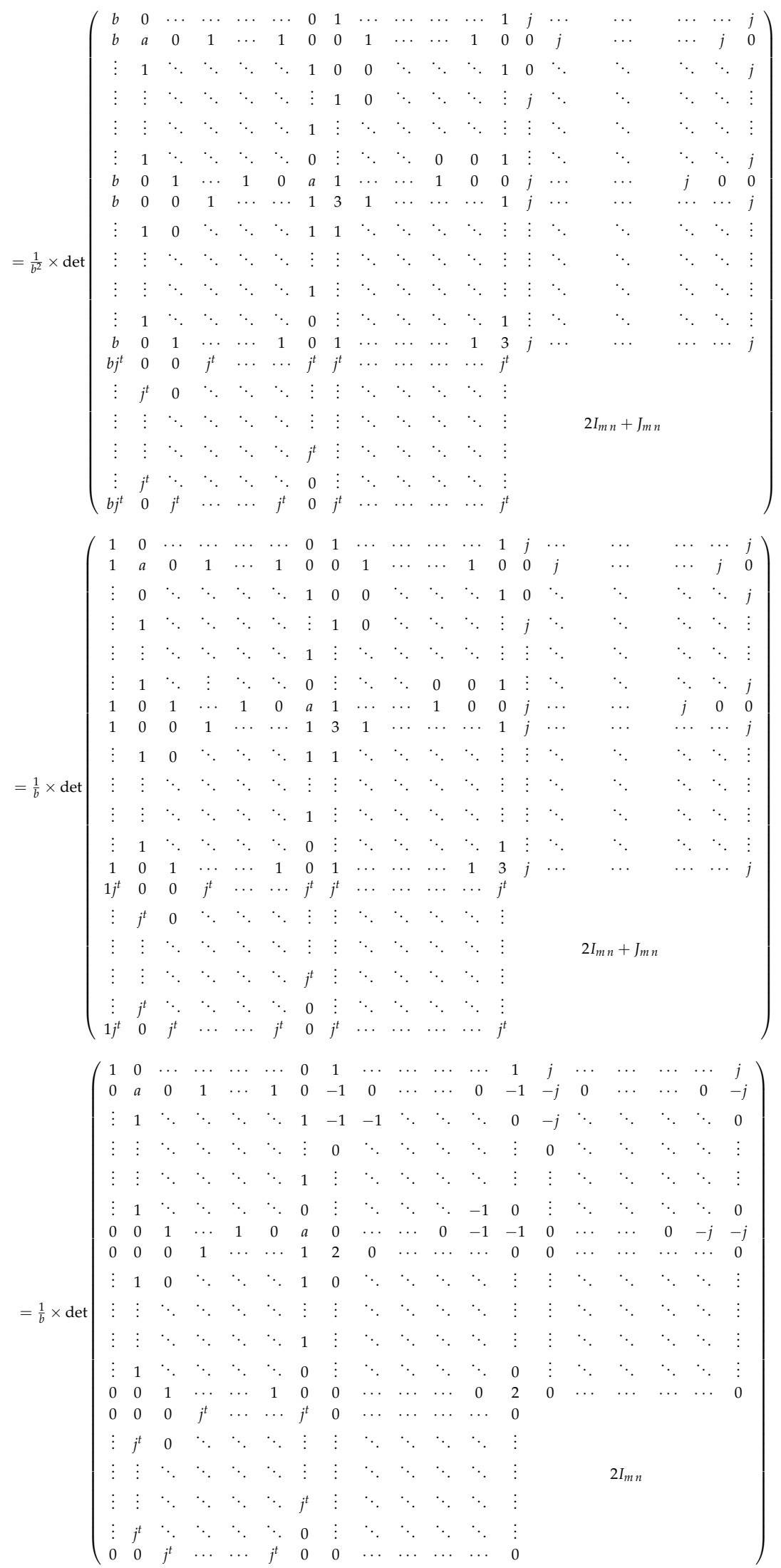


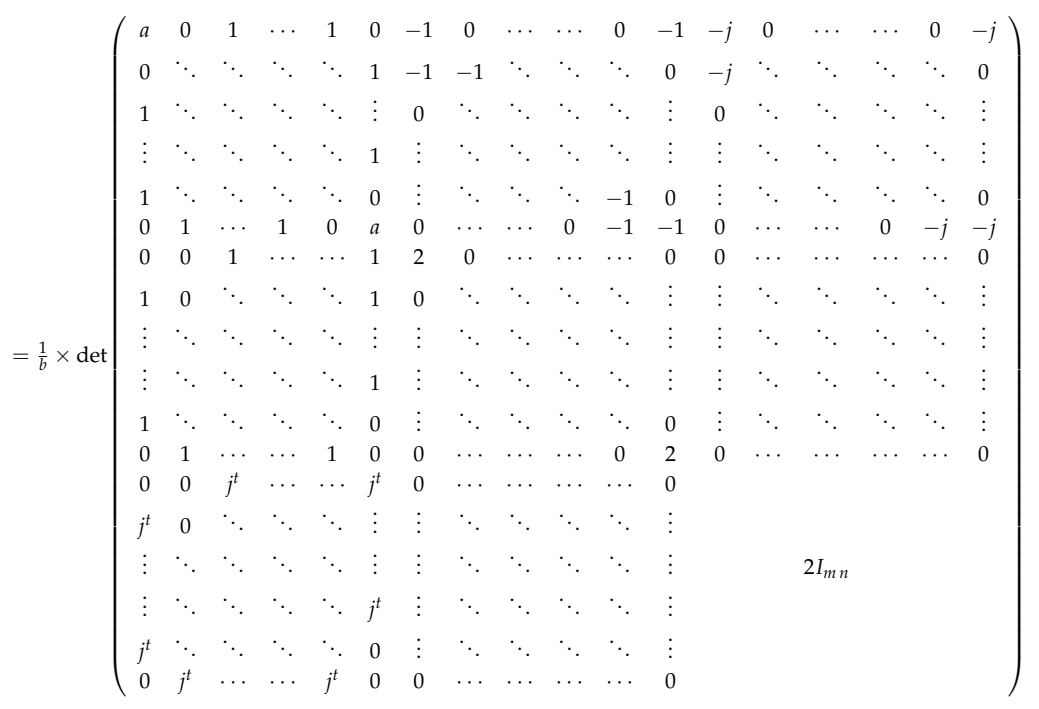

Using Lemma 3, yields

$$
\begin{aligned}
& \tau\left(C_{n}^{(m)}\right)=\frac{1}{b} \times \operatorname{det}\left(\begin{array}{cc}
A & B \\
C & 2 I_{m n}
\end{array}\right)=\frac{1}{b} \times \operatorname{det}\left(A-B \frac{1}{2 I_{m n}} C\right) \times 2^{m n} \\
& =\frac{1}{b} \times 2^{m n} \times 2^{-2 m} \times \operatorname{det}\left(\begin{array}{cccccccccccc}
2 a & n & 2(n+1) & \cdots & 2(n+1) & n & -2 & 0 & \cdots & \cdots & 0 & -2 \\
n & 2 a & n+2 & 2(n+1) & \cdots & 2(n+1) & -2 & \ddots & \ddots & \ddots & \ddots & 0 \\
2(n+1) & n & \ddots & \ddots & \ddots & \vdots & 0 & \ddots & \ddots & \ddots & \ddots & \vdots \\
\vdots & \ddots & \ddots & \ddots & \ddots & 2(n+1) & \vdots & \ddots & \ddots & \ddots & \ddots & \vdots \\
2(n+1) & \ddots & \ddots & \ddots & \ddots & n & \vdots & \ddots & \ddots & \ddots & \ddots & 0 \\
n & 2(n+1) & \cdots & 2(n+1) & n & 2 a & 0 & \cdots & \cdots & 0 & -2 & -2 \\
0 & 0 & 2 & \cdots & \cdots & 2 & 4 & 0 & \cdots & \cdots & \cdots & 0 \\
2 & \ddots & \ddots & \ddots & \ddots & \vdots & 0 & \ddots & \ddots & \ddots & \ddots & \vdots \\
\vdots & \ddots & \ddots & \ddots & \ddots & \vdots & \vdots & \ddots & \ddots & \ddots & \ddots & \vdots \\
\vdots & \ddots & \ddots & \ddots & \ddots & 2 & \vdots & \ddots & \ddots & \ddots & \ddots & \vdots \\
2 & \ddots & \ddots & \ddots & \ddots & 0 & \vdots & \ddots & \ddots & \ddots & \ddots & 0 \\
0 & 2 & \cdots & \cdots & 2 & 0 & 0 & \cdots & \cdots & \cdots & 0 & 4
\end{array}\right)
\end{aligned}
$$

Using Lemma 3 again, yields

$$
\begin{gathered}
\tau\left(C_{n}^{(m)}\right)=\frac{2^{m n-2 m}}{b} \times \operatorname{det}\left(\begin{array}{cc}
D & E \\
F & 4 I_{m}
\end{array}\right)=\frac{2^{m n}}{b} \times \operatorname{det}\left(D-E \frac{1}{4 I_{m}} F\right) \\
\tau\left(C_{n}^{(m)}\right)=\frac{2^{m n}}{b} \times \operatorname{det}\left(\begin{array}{cccccc}
2 a & (n+1) & 2(n+2) & \cdots & 2(n+2) & (n+1) \\
(n+1) & 2 a & (n+3) & \ddots & \ldots & 2(n+2) \\
2(n+2) & \ddots & \ddots & \ddots & \ddots & \vdots \\
\vdots & \ddots & \ddots & \ddots & \ddots & 2(n+2) \\
2(n+2) & \ddots & \ddots & \ddots & \ddots & (n+1) \\
(n+1) & 2(n+2) & \cdots & 2(n+2) & (n+1) & 2 a
\end{array}\right)
\end{gathered}
$$

Using properties of determinants, we have: 


$$
\begin{aligned}
& \tau\left(C_{n}^{(m)}\right)=\frac{2^{m n}}{b} \times \frac{2 b}{m n+3 m+2} \times \operatorname{det}\left(\begin{array}{cccccc}
(2 a-n-1) & 0 & (n+3) & \cdots & (n+3) & 0 \\
0 & (2 a-n-1) & 0 & \ddots & \ldots & (n+3) \\
(n+3) & \ddots & \ddots & \ddots & \ddots & \vdots \\
\vdots & \ddots & \ddots & \ddots & \ddots & (n+3) \\
(n+3) & \ddots & \ddots & \ddots & \ddots & 0 \\
0 & (n+3) & \cdots & (n+3) & 0 & (2 a-n-1)
\end{array}\right) \\
& =\frac{2^{m n+1}(n+3)^{m}}{m n+3 m+2} \times \operatorname{det}\left(\begin{array}{cccccc}
\frac{(2 a-n-1)}{(n+3)} & 0 & 1 & \cdots & 1 & 0 \\
0 & \frac{(2 a-n-1)}{(n+3)} & 0 & \ddots & \ddots & 1 \\
1 & \ddots & \ddots & \ddots & \ddots & \vdots \\
\vdots & \ddots & \ddots & \ddots & \ddots & 1 \\
1 & \ddots & \ddots & \ddots & \ddots & 0 \\
0 & 1 & \cdots & 1 & 0 & \frac{(2 a-n-1)}{(n+3)}
\end{array}\right)
\end{aligned}
$$

Using Lemma 2, yields:

$$
\begin{gathered}
\tau\left(C_{n}^{(m)}\right)=2^{m n+1} \times \frac{(n+3)^{m}}{m n+3 m+2} \times \frac{2\left(\frac{2 a-n-1}{n+3}+m-3\right)}{\frac{2 a-n-1}{n+3}-3} \times\left[T_{m}\left(\frac{\frac{2 a-n-1}{n+3}-1}{2}\right)-1\right] \\
=2^{m n+1} \times(n+3)^{m} \times\left[T_{m}\left(\frac{n+4}{n+3}\right)-1\right] .
\end{gathered}
$$

Using Equation (11), yields the result.

Definition 4. The pyramid graph $D_{n}^{(m)}$ is the graph created from the gear graph $G_{m+1}$ with vertices $\left\{u_{0} ; u_{1}, u_{2}, \ldots, u_{m} ; w_{1}, w_{2}, \ldots, w_{m}\right\}$ with double internal and external edges and $m$ sets of vertices, say, $\left\{v_{1}^{1}, v_{2}^{1}, \ldots, v_{n}^{1}\right\},\left\{v_{1}^{2}, v_{2}^{2}, \ldots, v_{n}^{2}\right\}, \ldots,\left\{v_{1}^{m}, v_{2}^{m}, \ldots, v_{n}^{m}\right\}$, such that for all $i=1,2, \ldots, n$ the vertex $v_{i}^{j}$ is adjacent to $u_{j}$ and $u_{j+1}$, where $j=1,2, \ldots, m-1$, and $v_{i}^{m}$ is adjacent to $u_{1}$ and $u_{m}$. See Figure 4.

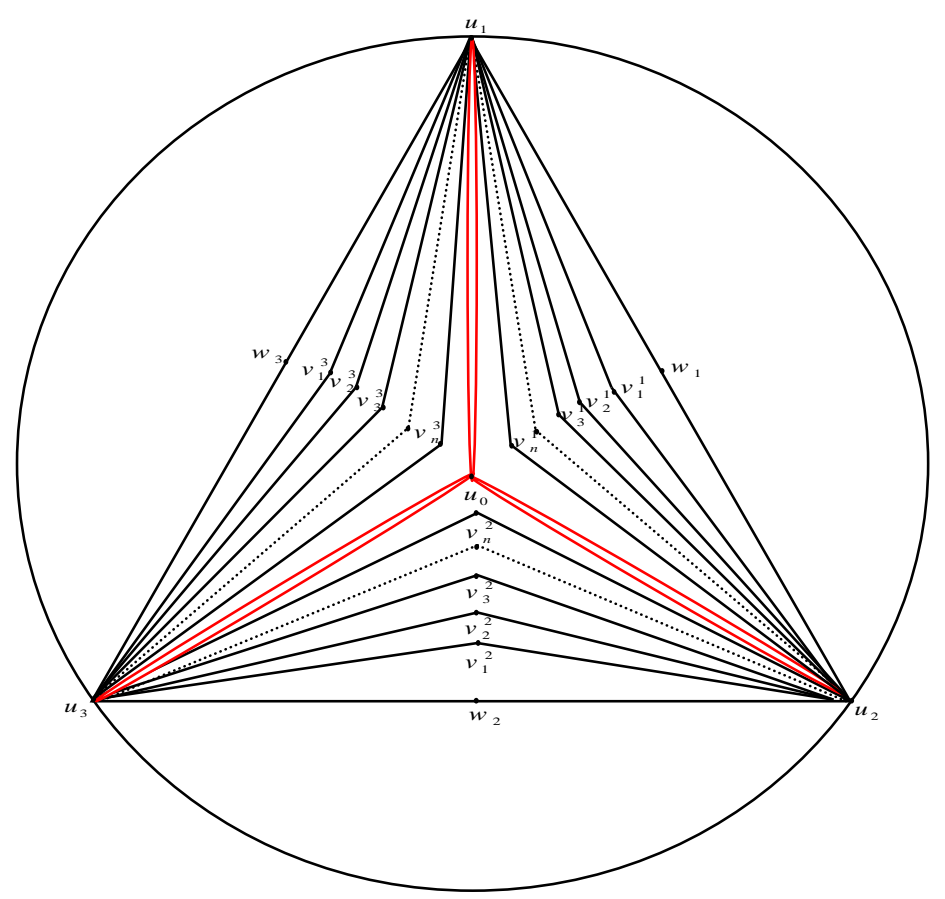

Figure 4. The pyramid graph $D_{n}^{(3)}$. 
Theorem 4. For $n \geq 0, m \geq 3, \tau\left(D_{n}^{(m)}\right)=2^{m n}\left[(n+5+2 \sqrt{n+4})^{m}+(n+5-2 \sqrt{n+4})^{m}-\right.$ $\left.2(n+3)^{m}\right]$.

Proof. Applying Lemma 1, we have:

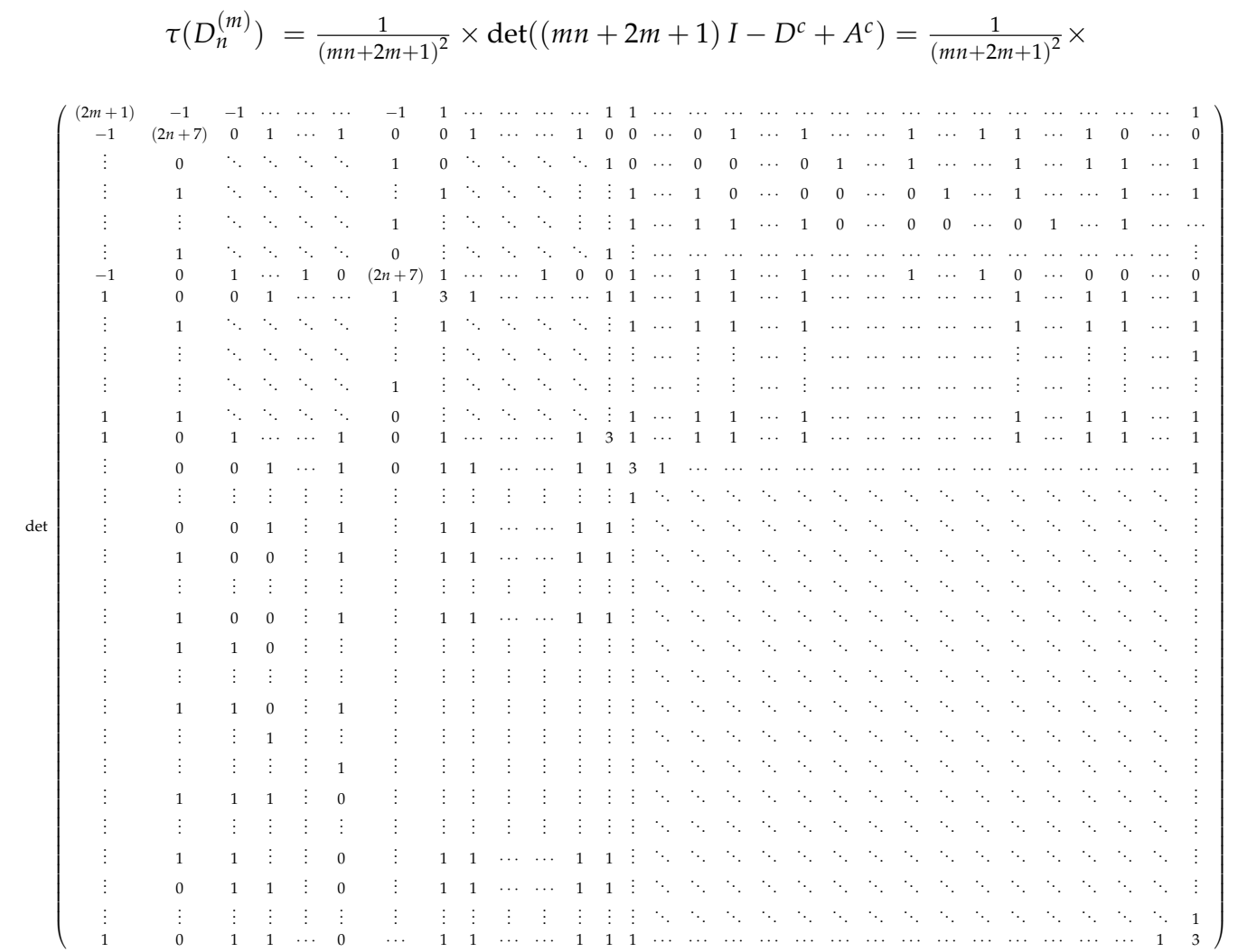

Let $j=(1 \cdots 1)$ be the $1 \times n$ matrix with all one, and $J_{n}$ the $n \times n$ matrix with all one. Set $a=2 n+7$ and $b=m n+2 m+1$. Then we have:

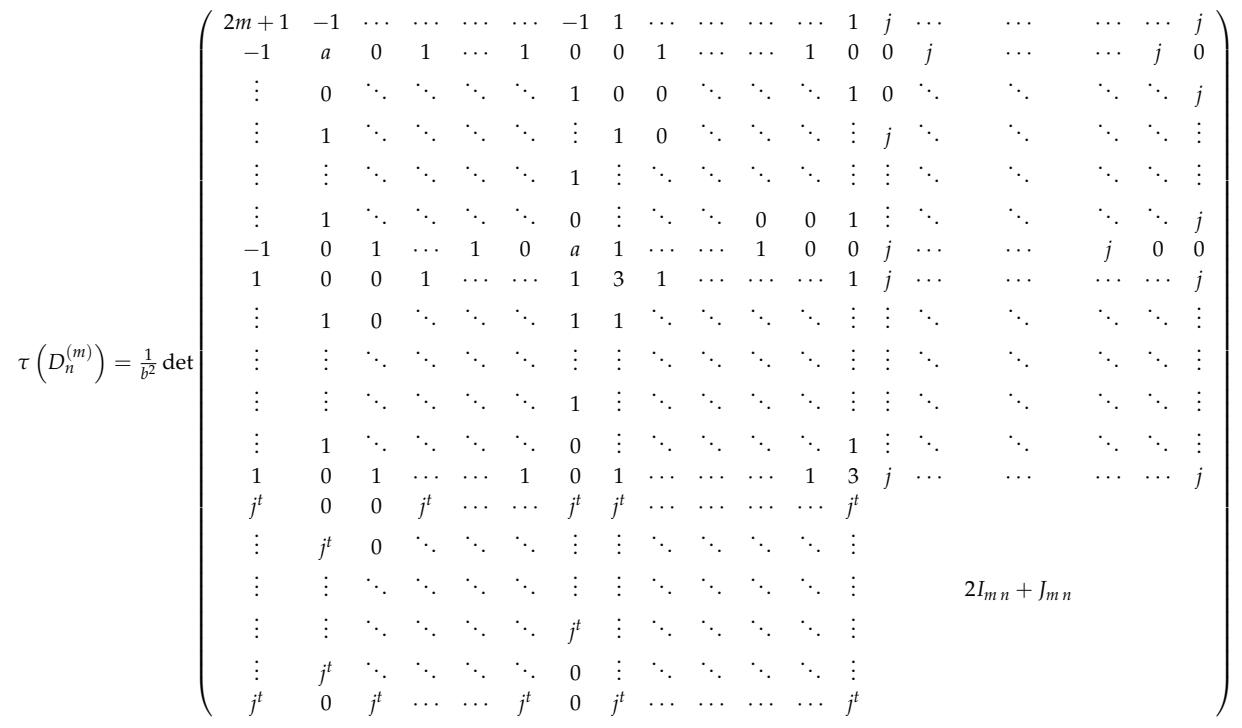




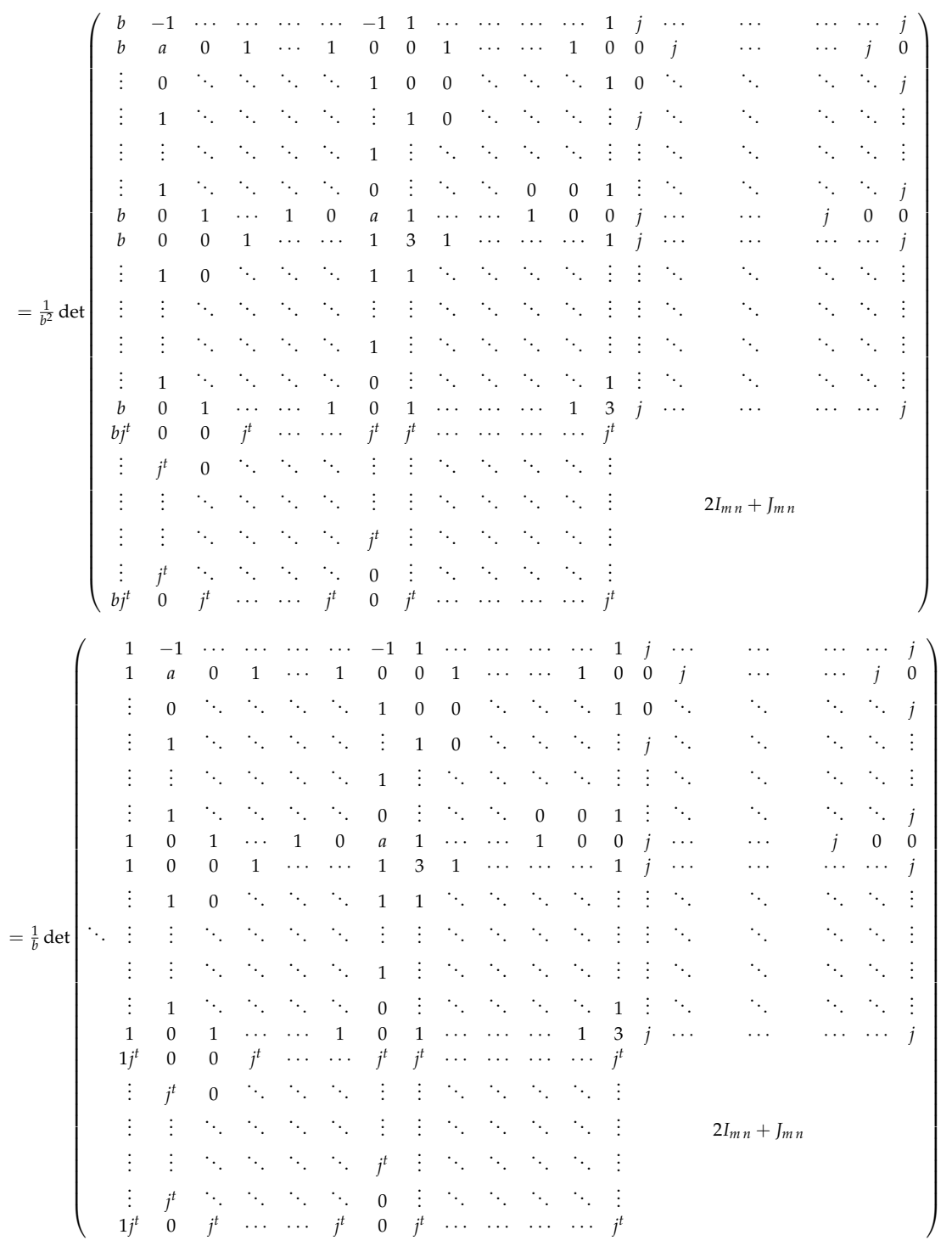

$$
=\frac{1}{b} \operatorname{det}\left(\begin{array}{ccccccccccccccccccc}
1 & -1 & \cdots & \cdots & \cdots & \cdots & -1 & 1 & \cdots & \cdots & \cdots & \cdots & 1 & j & \cdots & \cdots & \cdots & \cdots & j \\
0 & (a+1) & 1 & 2 & \cdots & 2 & 1 & -1 & 0 & \cdots & \cdots & 0 & -1 & -j & 0 & \cdots & \cdots & 0 & -j \\
\vdots & 1 & \ddots & \ddots & \ddots & \ddots & 2 & -1 & -1 & \ddots & \ddots & \ddots & 0 & -j & \ddots & \ddots & \ddots & \ddots & 0 \\
\vdots & 2 & \ddots & \ddots & \ddots & \ddots & \vdots & 0 & \ddots & \ddots & \ddots & \ddots & \vdots & 0 & \ddots & \ddots & \ddots & \ddots & \vdots \\
\vdots & \vdots & \ddots & \ddots & \ddots & \ddots & 2 & \vdots & \ddots & \ddots & \ddots & \ddots & \vdots & \vdots & \ddots & \ddots & \ddots & \ddots & \vdots \\
\vdots & 2 & \ddots & \ddots & \ddots & \ddots & 1 & \vdots & \ddots & \ddots & \ddots & -1 & 0 & \vdots & \ddots & \ddots & \ddots & \ddots & 0 \\
0 & 1 & 2 & \cdots & 2 & 1 & (a+1) & 0 & \cdots & \cdots & 0 & -1 & -1 & 0 & \cdots & \cdots & 0 & -j & -j \\
0 & 1 & 1 & 2 & \cdots & \cdots & 2 & 2 & 0 & \cdots & \cdots & \cdots & 0 & 0 & \cdots & \cdots & \cdots & \cdots & 0 \\
\vdots & 2 & 1 & \ddots & \ddots & \ddots & 2 & 0 & \ddots & \ddots & \ddots & \ddots & \vdots & \vdots & \ddots & \ddots & \ddots & \ddots & \vdots \\
\vdots & \vdots & \ddots & \ddots & \ddots & \ddots & \vdots & \vdots & \ddots & \ddots & \ddots & \ddots & \vdots & \vdots & \ddots & \ddots & \ddots & \ddots & \vdots \\
\vdots & \vdots & \ddots & \ddots & \ddots & \ddots & 2 & \vdots & \ddots & \ddots & \ddots & \ddots & \vdots & \vdots & \ddots & \ddots & \ddots & \ddots & \vdots \\
\vdots & 2 & \ddots & \ddots & \ddots & \ddots & 1 & \vdots & \ddots & \ddots & \ddots & \ddots & 0 & \vdots & \ddots & \ddots & \ddots & \ddots & \vdots \\
0 & 1 & 2 & \cdots & \cdots & 2 & 1 & 0 & \cdots & \cdots & \cdots & 0 & 2 & 0 & \cdots & \cdots & \cdots & \cdots & 0 \\
0 & j^{t} & j^{t} & 2 j^{t} & \cdots & \cdots & 2 j^{t} & 0 & \cdots & \cdots & \cdots & \cdots & 0 & & & & & \\
\vdots & 2 j^{t} & j^{t} & \ddots & \ddots & \ddots & \vdots & \vdots & \ddots & \ddots & \ddots & \ddots & \vdots & & & & & \\
\vdots & \vdots & \ddots & \ddots & \ddots & \ddots & \vdots & \vdots & \ddots & \ddots & \ddots & \ddots & \vdots & & & 2 I_{m n} & & & \\
\vdots & \vdots & \ddots & \ddots & \ddots & \ddots & 2 j^{t} & \vdots & \ddots & \ddots & \ddots & \ddots & \vdots & & & & & & \\
\vdots & 2 j^{t} & \ddots & \ddots & \ddots & \ddots & j^{t} & \vdots & \ddots & \ddots & \ddots & \ddots & \vdots & & & & & \\
0 & j^{t} & 2 j^{t} & \cdots & \cdots & 2 j^{t} & j^{t} & 0 & \cdots & \cdots & \cdots & \cdots & 0 & & & & &
\end{array}\right)
$$




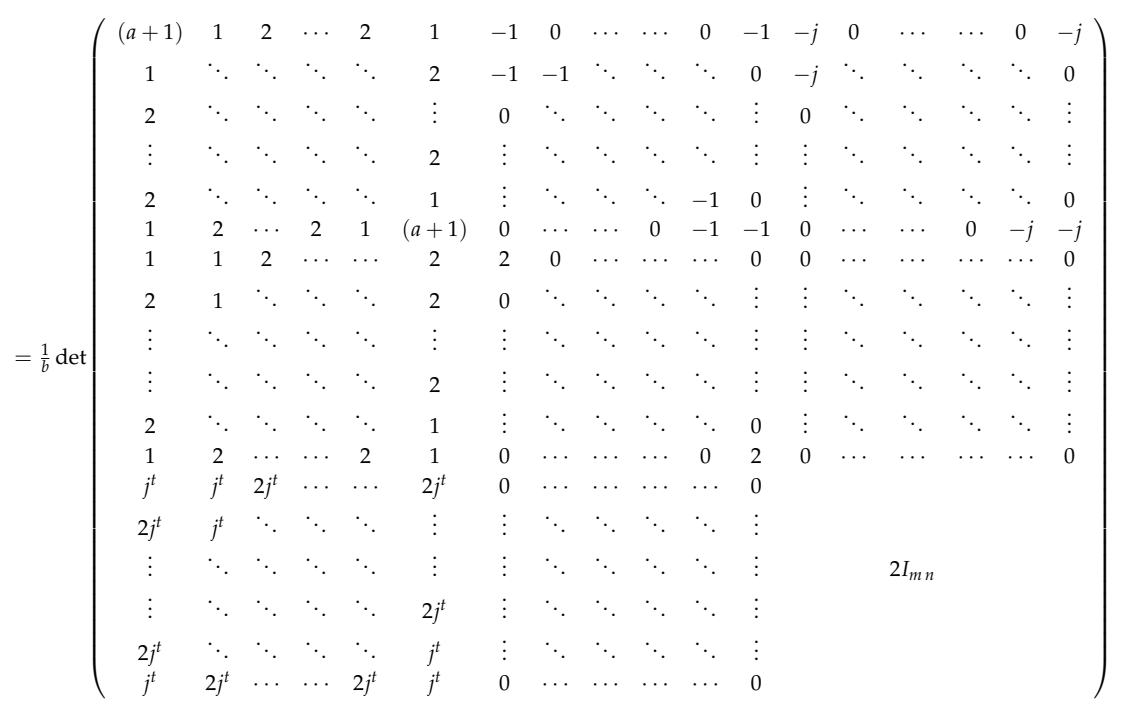

Using Lemma 3, yields

$$
\begin{aligned}
& \tau\left(D_{n}^{(m)}\right)=\frac{1}{b} \times \operatorname{det}\left(\begin{array}{cc}
A & B \\
C & 2 I_{m n}
\end{array}\right)=\frac{1}{b} \times \operatorname{det}\left(A-B \frac{1}{2 I_{m n}} C\right) \times 2^{m n} \\
& =\frac{1}{b} 2^{m n} \times 2^{-2 m} \times \operatorname{det}\left(\begin{array}{cccccccccccc}
(2 a+2 n+2) & 3 n+2 & 4(n+1) & \cdots & 4(n+1) & 3 n+2 & -2 & 0 & \cdots & \cdots & 0 & -2 \\
3 n+2 & (2 a+2 n+2) & 3 n+2 & 4(n+1) & \cdots & 4(n+1) & -2 & \ddots & \ddots & \ddots & \ddots & 0 \\
4(n+1) & 3 n+4 & \ddots & \ddots & \ddots & \vdots & 0 & \ddots & \ddots & \ddots & \ddots & \vdots \\
\vdots & \ddots & \ddots & \ddots & \ddots & 4(n+1) & \vdots & \ddots & \ddots & \ddots & \ddots & \vdots \\
4(n+1) & \ddots & \ddots & \ddots & \ddots & 3 n+2 & \vdots & \ddots & \ddots & \ddots & \ddots & 0 \\
3 n+2 & 4(n+1) & \cdots & 4(n+1) & 3 n+2 & (2 a+2 n+2) & 0 & \cdots & \cdots & 0 & -2 & -2 \\
2 & 2 & 4 & \cdots & \cdots & 4 & 4 & 0 & \cdots & \cdots & \cdots & 0 \\
4 & \ddots & \ddots & \ddots & \ddots & \vdots & 0 & \ddots & \ddots & \ddots & \ddots & \vdots \\
\vdots & \ddots & \ddots & \ddots & \ddots & \vdots & \vdots & \ddots & \ddots & \ddots & \ddots & \vdots \\
\vdots & \ddots & \ddots & \ddots & \ddots & 4 & \vdots & \ddots & \ddots & \ddots & \ddots & \vdots \\
4 & \ddots & \ddots & \ddots & \ddots & 2 & \vdots & \ddots & \ddots & \ddots & \ddots & 0 \\
2 & 4 & \cdots & \cdots & 4 & 2 & 0 & \cdots & \cdots & \cdots & 0 & 4
\end{array}\right)
\end{aligned}
$$

Using Lemma 3, yields

$$
\begin{aligned}
& \tau\left(D_{n}^{(m)}\right)=\frac{2^{m n-2 m}}{b} \times \operatorname{det}\left(\begin{array}{cc}
A & B \\
C & 4 I_{m}
\end{array}\right)=\frac{2^{m n}}{b} \times \operatorname{det}\left(A-B \frac{1}{4 I_{m}} C\right) \\
& \tau\left(D_{n}^{(m)}\right)=\frac{2^{m n}}{b} \times \operatorname{det}\left(\begin{array}{cccccc}
(2 a+2 n+4) & (3 n+5) & 4(n+2) & \ldots & 4(n+2) & (3 n+5) \\
(3 n+5) & (2 a+2 n+4) & (3 n+5) & \ddots & \ldots & 4(n+2) \\
4(n+2) & \ddots & \ddots & \ddots & \ddots & \vdots \\
\vdots & \ddots & \ddots & \ddots & \ddots & 4(n+2) \\
4(n+2) & \ddots & \ddots & \ddots & \ddots & (3 n+5) \\
(3 n+5) & 4(n+2) & \ldots & 4(n+2) & (3 n+5) & (2 a+2 n+4)
\end{array}\right)
\end{aligned}
$$

Straightforward inducement using properties of determinants, we get:

$$
\tau\left(D_{n}^{(m)}\right)=\frac{2^{m n}}{b} \times \frac{4 b}{m n+3 m+4} \times \operatorname{det}\left(\begin{array}{cccccc}
(2 a-n-1) & 0 & (n+3) & \cdots & (n+3) & 0 \\
0 & (2 a-n-1) & 0 & \ddots & \cdots & (n+3) \\
(n+3) & \ddots & \ddots & \ddots & \ddots & \vdots \\
\vdots & \ddots & \ddots & \ddots & \ddots & (n+3) \\
(n+3) & \ddots & \ddots & \ddots & \ddots & 0 \\
0 & (n+3) & \cdots & (n+3) & 0 & (2 a-n-1)
\end{array}\right)
$$




$$
=\frac{2^{m n+2}(n+3)^{m}}{m n+3 m+4} \times \operatorname{det}\left(\begin{array}{cccccc}
\frac{(2 a-n-1)}{(n+3)} & 0 & 1 & \cdots & 1 & 0 \\
0 & \frac{(2 a-n-1)}{(n+3)} & 0 & \ddots & \ddots & 1 \\
1 & \ddots & \ddots & \ddots & \ddots & \vdots \\
\vdots & \ddots & \ddots & \ddots & \ddots & 1 \\
1 & \ddots & \ddots & \ddots & \ddots & 0 \\
0 & 1 & \cdots & 1 & 0 & \frac{(2 a-n-1)}{(n+3)}
\end{array}\right)
$$

Using Lemma 2, yields:

$$
\tau\left(D_{n}^{(m)}\right)=2^{m n+2} \times \frac{(n+3)^{m}}{m n+3 m+4} \times \frac{2\left(\frac{2 a-n-1}{n+3}+m-3\right)}{\frac{2 a-n-1}{n+3}-3} \times\left[T_{m}\left(\frac{\frac{2 a-n-1}{n+3}-1}{2}\right)-1\right]=2^{m n+1} \times(n+3)^{m} \times\left[T_{m}\left(\frac{n+5}{n+3}\right)-1\right] .
$$

Using Equation (11), yields the result.

\section{Numerical Results}

The following Table 1 illustrates some values of the number of spanning trees of studied pyramid graphs.

Table 1. Some values of the number of spanning trees of studied pyramid graphs.

\begin{tabular}{cccccc}
\hline$m$ & $n$ & $\tau\left(P_{n}^{(m)}\right)$ & $\tau\left(A_{n}^{(m)}\right)$ & $\tau\left(B_{n}^{(m)}\right)$ & $\tau\left(C_{n}^{(m)}\right)$ \\
\hline 3 & 0 & 50 & 196 & 242 & 676 \\
3 & 1 & 1024 & 3200 & 3136 & 8192 \\
3 & 2 & 15,488 & 43,264 & 36,992 & 92,416 \\
3 & 3 & 200,704 & 524,288 & 409,600 & 991,232 \\
3 & 4 & $2,367,488$ & $5,914,624$ & $4,333,568$ & $10,240,000$ \\
3 & 5 & $26,214,400$ & $63,438,848$ & $44,302,336$ & $102,760,448$ \\
4 & 0 & 192 & 1152 & 1792 & 6400 \\
4 & 1 & 11,520 & 49,152 & 57,600 & 184,320 \\
4 & 2 & 458,752 & $1,638,400$ & $1,622,016$ & $4,816,896$ \\
4 & 3 & $14,745,600$ & $47,185,920$ & $41,746,432$ & $117,440,512$ \\
4 & 4 & $415,236,096$ & $1,233,125,376$ & $1,006,632,960$ & $2,717,908,992$ \\
4 & 5 & $10,687,086,592$ & $30,064,771,072$ & $23,102,226,432$ & $60,397,977,600$ \\
5 & 0 & 722 & 6724 & 12,482 & 58,564 \\
5 & 1 & 123,904 & 739,328 & 984,064 & $3,964,928$ \\
5 & 2 & $12,781,568$ & $59,969,536$ & $65,619,968$ & $237,899,776$ \\
5 & 3 & $1,007,681,536$ & $4,060,086,272$ & $3,901,751,296$ & $13,088,325,632$ \\
5 & 4 & $67,194,847,232$ & $243,609,370,624$ & $213,408,284,672$ & $674,448,277,504$ \\
5 & 5 & $3,995,393,327,104$ & $243,609,370,624$ & $10,953,240,346,624$ & $33,019,708,571,648$ \\
\hline
\end{tabular}

\section{Conclusions}

The number of spanning trees $\tau(G)$ in graphs (networks) is an important invariant. The computation of this number is not only beneficial from a mathematical (computational) standpoint, but it is also an important measure of the reliability of a network and electrical circuit layout. Some computationally laborious problems such as the traveling salesman problem can be resolved approximately by using spanning trees. In this paper, we define some classes of pyramid graphs created by a gear graph and we have studied the problem of computing the number of spanning trees of these graphs.

Author Contributions: All authors contributed equally to this work. Funding Acquisition, J.-B.L.; Methodology, J.-B.L. and S.N.D. Daoud; Writing—Original Draft, J.-B.L. and S.N.D. Daoud; All authors read and approved the final manuscript. 
Funding: The work was partially supported by the China Postdoctoral Science Foundation under grant No. 2017M621579 and the Postdoctoral Science Foundation of Jiangsu Province under grant No. 1701081B, Project of Anhui Jianzhu University under Grant no. 2016QD116 and 2017dc03, Anhui Province Key Laboratory of Intelligent Building \& Building Energy Saving.

Acknowledgments: The authors are grateful to the anonymous reviewers for their helpful comments and suggestions for improving the original version of the paper.

Conflicts of Interest: The authors declare that there are no conflicts of interest regarding the publication of this paper.

\section{References}

1. Applegate, D.L.; Bixby, R.E.; Chvátal, V.; Cook, W.J. The Traveling Salesman Problem: A Computational Study; Princeton University Press: Princeton, NJ, USA, 2006.

2. Cvetkoviě, D.; Doob, M.; Sachs, H. Spectra of Graphs: Theory and Applications, 3rd ed.; Johann Ambrosius Barth: Heidelberg, Germany, 1995.

3. Kirby, E.C.; Klein, D.J.; Mallion, R.B.; Pollak, P.; Sachs, H. A theorem for counting spanning trees in general chemical graphs and its particular application to toroidal fullerenes. Croat. Chem. Acta 2004, 77, 263-278.

4. Boesch, F.T.; Satyanarayana, A.; Suffel, C.L. A survey of some network reliability analysis and synthesis results. Networks 2009, 54, 99-107. [CrossRef]

5. Boesch, F.T. On unreliability polynomials and graph connectivity in reliable network synthesis. J. Graph Theory 1986, 10, 339-352. [CrossRef]

6. Wu, F.Y. Number of spanning trees on a Lattice. J. Phys. A 1977, 10, 113-115. [CrossRef]

7. Zhang, F.; Yong, X. Asymptotic enumeration theorems for the number of spanning trees and Eulerian trail in circulant digraphs \& graphs. Sci. China Ser. A 1999, 43, 264-271.

8. Chen, G.; Wu, B.; Zhang, Z. Properties and applications of Laplacian spectra for Koch networks. J. Phys. A Math. Theor. 2012, 45, 025102.

9. Atajan, T.; Inaba, H. Network reliability analysis by counting the number of spanning trees. In Proceedings of the IEEE International Symposium on Communications and Information Technology, ISCIT 2004, Sapporo, Japan, 26-29 October 2004; pp. 601-604.

10. Brown, T.J.N.; Mallion, R.B.; Pollak, P.; Roth, A. Some methods for counting the spanning trees in labelled molecular graphs, examined in relation to certain fullerenes. Discret. Appl. Math. 1996, 67, 51-66. [CrossRef]

11. Kirchhoff, G.G. Uber die Auflosung der Gleichungen, auf welche man be ider Untersuchung der Linearen Verteilung galvanischer Storme gefuhrt wird. Ann. Phys. Chem. 1847, 72, 497-508. [CrossRef]

12. Kelmans, A.K.; Chelnokov, V.M. A certain polynomials of a graph and graphs with an extermal number of trees. J. Comb. Theory B 1974, 16, 197-214. [CrossRef]

13. Biggs, N.L. Algebraic Graph Theory, 2nd ed.; Cambridge University Press: Cambridge, UK, 1993; p. 205.

14. Daoud, S.N. The deletion-contraction method for counting the number of spanning trees of graphs. Eur. Phys. J. Plus 2015, 130, 217. [CrossRef]

15. Shang, Y. On the number of spanning trees, the Laplacian eigenvalues, and the Laplacian Estrada index of subdivided-line graphs. Open Math. 2016, 14, 641-648. [CrossRef]

16. Bozkurt, Ş.B.; Bozkurt, D. On the Number of Spanning Trees of Graphs. Sci. World J. 2014, $2014,294038$. [CrossRef] [PubMed]

17. Daoud, S.N. Number of Spanning Trees in Different Product of Complete and Complete Tripartite Graphs. ARS Comb. 2018, 139, 85-103.

18. Daoud, S.N. Complexity of Graphs Generated by Wheel Graph and Their Asymptotic Limits. J. Egypt. Math. Soc. 2017, 25, 424-433. [CrossRef]

19. Daoud, S.N. Chebyshev polynomials and spanning tree formulas. Int. J. Math. Comb. 2012, 4, 68-79.

20. Zhang, Y.; Yong, X.; Golin, M.J. Chebyshev polynomials and spanning trees formulas for circulant and related graphs. Discret. Math. 2005, 298, 334-364. [CrossRef] 
21. Daoud, S.N. On a class of some pyramid graphs and Chebyshev polynomials. J. Math. Probl. Eng. Hindawi Publ. Corp. 2013, 2013, 820549.

22. Marcus, M. A Survey of Matrix Theory and Matrix Inequalities; University Allyn and Bacon. Inc.: Boston, MA, USA, 1964. 